Supporting Information for:

\title{
A Dramatic Effect of Lewis Acid Catalyst on Nickel-catalyzed Carbocyanation of Alkynes
}

\author{
Yoshiaki Nakao,* Akira Yada, Shiro Ebata, and Tamejiro Hiyama* \\ Department of Material Chemistry, Graduate School of Engineering, \\ Kyoto University, Kyoto 615-8510 Japan
}

General. All manipulations of oxygen- and moisture-sensitive materials were conducted with a standard Schlenk technique or in a dry box under an argon atmosphere. Flash column chromatography was performed using Kanto Chemical silica gel (spherical, 40-50 $\mu \mathrm{m}$ ). Analytical thin layer chromatography (TLC) was performed on Merck Kieselgel $60 \mathrm{~F}_{254}(0.25 \mathrm{~mm})$ plates. Visualization was accomplished with UV light $(254 \mathrm{~nm})$ and/or an aqueous alkaline $\mathrm{KMnO}_{4}$ solution followed by heating.

Apparatus. Proton and carbon nuclear magnetic resonance spectra $\left({ }^{1} \mathrm{H}\right.$ NMR and ${ }^{13} \mathrm{C}$ NMR $)$ were recorded on a Varian Mercury $400\left({ }^{1} \mathrm{H}\right.$ NMR, $400 \mathrm{MHz} ;{ }^{13} \mathrm{C}$ NMR, $\left.101 \mathrm{MHz}\right)$ spectrometer with solvent resonance as the internal standard ( ${ }^{1} \mathrm{H} \mathrm{NMR}, \mathrm{CHCl}_{3}$ at $7.26 \mathrm{ppm} ;{ }^{13} \mathrm{C} \mathrm{NMR} \mathrm{CDCl}_{3}$ at 77.0 ppm). ${ }^{1} \mathrm{H}$ NMR data are reported as follows: chemical shift, multiplicity ( $\mathrm{s}=$ singlet, $\mathrm{d}=$ doublet, $\mathrm{t}=$ triplet, $\mathrm{q}=$ quartet, quint $=$ quintet, sext $=$ sextet, $\mathrm{br}=$ broad, $\mathrm{m}=$ multiplet $)$, coupling constants $(\mathrm{Hz})$, and integration. Melting points were determined using a YANAKO MP-500D. Mass spectra were obtained with a JEOL JMS-700 (EI). GC analysis was performed on a Shimadzu GC 2014 equipped with an ENV-1 column (Kanto Chemical, $30 \mathrm{~m} \times 0.25 \mathrm{~mm}$, pressure $=31.7 \mathrm{kPa}$, detector $=$ FID, $290{ }^{\circ} \mathrm{C}$ ) with helium gas as a carrier.

Chemicals. Unless otherwise noted, commercially available reagents were used without purification. Anhydrous toluene purchased from Kanto Chemical was degassed by purging vigorously with argon for $20 \mathrm{~min}$ and further purified by passage through activated alumina under positive argon pressure as described by Grubbs et al. ${ }^{1}$ 4-Cyanophenyl-[2-(tetrahydro- $2 \mathrm{H}$-pyranoxymethyl)phenyl]dimethylsilane (1c) ${ }^{2}$ 4-diphenylaminobenzonitrile (1e), ${ }^{3}$ 1-methyl-3-cyanoindole (1i), ${ }^{4}$ 1-(4-methoxyphenyl)-1-butyne (2c), ${ }^{5}$ dichlorobis(dimethylphenylphosphine)nickel(II), ${ }^{6}$ cyanomethylenecyclohexane (4d) ${ }^{7}$ 3,3-diphenyl-2-propenitrile (4e) ${ }^{8}$ and 1-octynyl(trimethyl)silane $(\mathbf{2 e})^{9}$ were prepared according 
to the respective literature procedure.

Nickel-LA-catalyzed arylcyanation of alkynes. A general procedure. An aryl cyanide (1.00 $\mathrm{mmol})$, a Lewis acid $(0.040 \mathrm{mmol})$, an alkyne $(1.00 \mathrm{mmol})$, and $\mathrm{C}_{12} \mathrm{H}_{26}$ (internal standard, $56 \mathrm{mg}, 0.33$ $\mathrm{mmol})$ were added sequentially to a solution of $\mathrm{Ni}(\mathrm{cod})_{2}(2.8 \mathrm{mg}, 0.010 \mathrm{mmol})$ and a ligand $(0.020$ $\mathrm{mmol})$ in toluene $(1.0 \mathrm{~mL})$ in a dry box. The vial was taken outside the dry box and heated at $50{ }^{\circ} \mathrm{C}$ for the time specified Table 1. The resulting mixture was filtered through a silica gel pad, concentrated in vacuo, and purified by flash silica gel column chromatography to give the corresponding arylcyanation products in yields listed in Table 1. The spectra of 3aa and 3ba agreed well with those reported by us previously. $^{10}$

Table S1. Optimization of a combination of a ligand and Lewis acid for the nickel-catalyzed addition of $1 \mathbf{a}$ across $\mathbf{2 a}$.
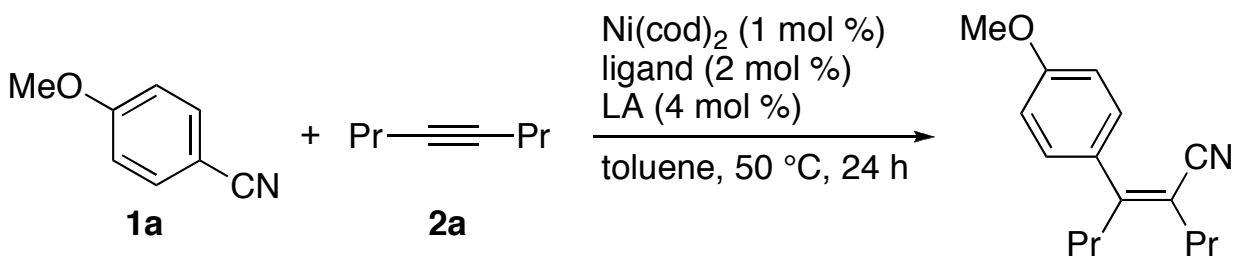

3aa

\begin{tabular}{lcccc}
\hline & \multicolumn{4}{c}{ Lewis acid $^{a} / \mathrm{GC}$ yield of 3aa $(\%)^{b}$} \\
\cline { 2 - 5 } & $\mathrm{BPh}_{3}$ & $\mathrm{AlMe}_{3}$ & $\mathrm{AlMe}_{2} \mathrm{Cl}$ & none \\
\hline $\mathrm{PMe}_{3}$ & 31 & 60 & 88 & 1 \\
$\mathrm{P}(n-\mathrm{Bu})_{3}$ & 39 & 63 & 41 & $<1$ \\
$\mathrm{PPhMe}_{2}$ & 78 & 95 & $>99$ & $<1$ \\
$\mathrm{PPh}_{2} \mathrm{Me}$ & 92 & 92 & 98 & $<1$ \\
$\mathrm{PPh}_{2} \mathrm{Cy}$ & 79 & 95 & 50 & $<1$ \\
$\mathrm{P}\left(4-\mathrm{MeO}-\mathrm{C}_{6} \mathrm{H}_{4}\right)_{3}$ & 53 & 29 & 6 & $<1$ \\
$\mathrm{Ph}_{2} \mathrm{P}\left(\mathrm{CH}_{2}\right)_{6} \mathrm{PPh}_{2}$ & 60 & 72 & 66 & $<1$ \\
\hline
\end{tabular}

${ }^{a}$ Other Lewis acids including $\mathrm{AlMeCl}_{2}, \mathrm{AlCl}_{3}, \mathrm{Al}(\mathrm{OR})_{3}(\mathrm{R}=\mathrm{Et}, i$-Pr, and $\mathrm{Ph})$, $\mathrm{AlPh}_{3}, \mathrm{~B}\left(\mathrm{C}_{6} \mathrm{~F}_{5}\right)_{3}, \mathrm{BEt}_{3}$, and $\mathrm{BF}_{3} \bullet \mathrm{OEt}_{2}$ were ineffective. ${ }^{b} \mathrm{GC}$ yields estimated using dodecane as an internal standard. 


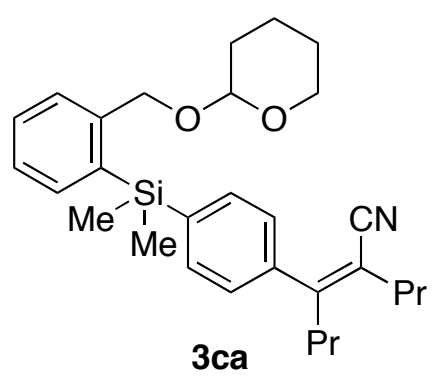

(Z)-3-(4-[2-(2-Tetrahydro-2H-pyranoxymethyl)phenyl]dimethylsilylphe nyl)-2-propyl-2-hexenenitrile (3ca). A colorless oil, $\mathrm{R}_{\mathrm{f}} 0.35$ (hexane-ethyl acetate $=10: 1) .{ }^{1} \mathrm{H}$ NMR $\left(400 \mathrm{MHz}, \mathrm{CDCl}_{3}\right) \delta 7.54(\mathrm{dd}, J=7.5,1.3 \mathrm{~Hz}$, $1 \mathrm{H}), 7.51-7.47(\mathrm{~m}, 3 \mathrm{H}), 7.41(\mathrm{td}, J=7.5,1.5 \mathrm{~Hz}, 1 \mathrm{H}), 7.33-7.23(\mathrm{~m}, 3 \mathrm{H})$, $4.67(\mathrm{~d}, J=12.1 \mathrm{~Hz}, 1 \mathrm{H}), 4.48(\mathrm{t}, J=3.5 \mathrm{~Hz}, 1 \mathrm{H}), 4.39(\mathrm{~d}, J=12.1 \mathrm{~Hz}, 1 \mathrm{H})$, 3.84-3.74 (m, 1H), 3.49-3.39 (m, 1H), $2.49(\mathrm{t}, J=7.8 \mathrm{~Hz}, 2 \mathrm{H}), 2.35$ (t, $J=$ $7.6 \mathrm{~Hz}, 2 \mathrm{H}), 1.83-1.40(\mathrm{~m}, 8 \mathrm{H}), 1.30$ (sext, $J=7.5 \mathrm{~Hz}, 2 \mathrm{H}), 1.01$ (t, $J=7.4 \mathrm{~Hz}, 3 \mathrm{H}), 0.87$ (t, $J=7.4 \mathrm{~Hz}$, $3 \mathrm{H}), 0.61(\mathrm{~s}, 3 \mathrm{H}), 0.60(\mathrm{~s}, 3 \mathrm{H}) ;{ }^{13} \mathrm{C} \mathrm{NMR}\left(101 \mathrm{MHz}, \mathrm{CDCl}_{3}\right) \delta$ 158.6, 144.2, 140.6, 139.5, 135.7, 135.5, 134.0, 129.7, 128.5, 127.0, 126.8, 119.6, 111.5, 97.8, 68.8, 62.0, 35.6, 32.5, 30.4, 25.4, 21.7, 21.1, 19.3, $13.8,13.5,-0.96,-1.11$; IR (neat): 2959, 2872, 2361, 2210, 1458, 1437, 1389, 1350, 1258, 1202, 1119 , 1078, 1028, 833, 814, 775, $756 \mathrm{~cm}^{-1}$; Anal. Calcd for $\mathrm{C}_{29} \mathrm{H}_{39} \mathrm{NO}_{2} \mathrm{Si}$; C, 75.44; H, 8.51. Found: C, 75.53; H, 8.69 .

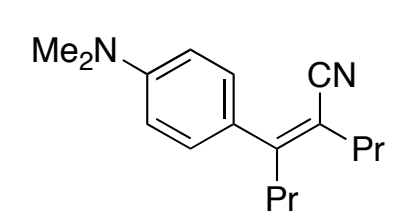

3da

(Z)-3-(4-N,N-Dimethylaminoyphenyl)-2-propyl-2-hexenenitrile (3da). A colorless oil, $\mathrm{R}_{\mathrm{f}} 0.61$ (hexane-ethyl acetate $\left.=2: 1\right) .{ }^{1} \mathrm{H} \mathrm{NMR}\left(400 \mathrm{MHz}, \mathrm{CDCl}_{3}\right)$ $\delta 7.26(\mathrm{~d}, J=9.0 \mathrm{~Hz}, 2 \mathrm{H}), 6.70(\mathrm{~d}, J=9.0 \mathrm{~Hz}, 2 \mathrm{H}), 2.98(\mathrm{~s}, 6 \mathrm{H}), 2.49(\mathrm{t}, J=$ $7.7 \mathrm{~Hz}, 2 \mathrm{H}), 2.33(\mathrm{t}, J=7.7 \mathrm{~Hz}, 2 \mathrm{H}), 1.66$ (sext, $J=7.5 \mathrm{~Hz}, 2 \mathrm{H}), 1.32$ (sext, $J=$ $7.5 \mathrm{~Hz}, 2 \mathrm{H}), 1.01(\mathrm{t}, J=7.3 \mathrm{~Hz}, 3 \mathrm{H}), 0.86(\mathrm{t}, J=7.4 \mathrm{~Hz}, 3 \mathrm{H}) ;{ }^{13} \mathrm{C}$ NMR $\left(101 \mathrm{MHz}, \mathrm{CDCl}_{3}\right) \delta 158.8$, 150.5, 128.9, 127.3, 120.7, 111.6, 108.9, 40.2, 35.3, 32.7, 21.9, 21.4, 13.8, 13.5; IR (neat): 2961, 2932, 2872, 2205, 1611, 1524, 1454, 1445, 1360, 1229, 1202, 1167, 947, 820, $733 \mathrm{~cm}^{-1}$; Anal. Calcd for $\mathrm{C}_{17} \mathrm{H}_{24} \mathrm{~N}_{2} ; \mathrm{C}, 79.64 ; \mathrm{H}, 9.44$. Found: C, 79.64; H, 9.50.

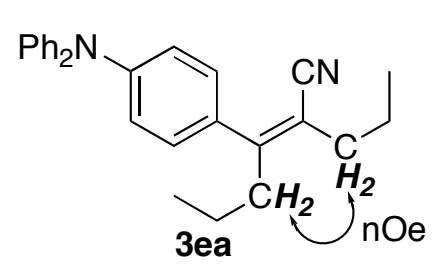

(Z)-3-(4-N,N-Diphenylaminoyphenyl)-2-propyl-2-hexenenitrile (3ea). A colorless oil, $\mathrm{R}_{\mathrm{f}} 0.24$ (hexane-ethyl acetate $\left.=10: 1\right) .{ }^{1} \mathrm{H}$ NMR $(400 \mathrm{MHz}$, $\left.\mathrm{CDCl}_{3}\right) \delta 7.32-7.00(\mathrm{~m}, 14 \mathrm{H}), 2.49(\mathrm{t}, J=7.7 \mathrm{~Hz}, 2 \mathrm{H}), 2.34(\mathrm{t}, J=7.6 \mathrm{~Hz}$, $2 \mathrm{H}), 1.67$ (sext, $J=7.5 \mathrm{~Hz}, 2 \mathrm{H}), 1.35$ (sext, $J=7.5 \mathrm{~Hz}, 2 \mathrm{H}), 1.01(\mathrm{t}, J=7.3$ $\mathrm{Hz}, 3 \mathrm{H}), 0.90(\mathrm{t}, J=7.4 \mathrm{~Hz}, 3 \mathrm{H}) ;{ }^{13} \mathrm{C} \mathrm{NMR}\left(101 \mathrm{MHz}, \mathrm{CDCl}_{3}\right) \delta 158.3,148.1,147.3,133.1,129.3$, 128.7, 125.0, 123.4, 122.0, 120.1, 110.5, 35.5, 32.7, 21.8, 21.3, 13.9, 13.5; IR (neat): 2963, 2932, 2872, 2208, 1591, 1506, 1493, 1327, 1277, 839, 754, $696 \mathrm{~cm}^{-1}$; HRMS (EI) Calcd for $\mathrm{C}_{27} \mathrm{H}_{28} \mathrm{~N}_{2}: \mathrm{M}^{+}$, 380.2252. Found: $m / z 380.2244$. 


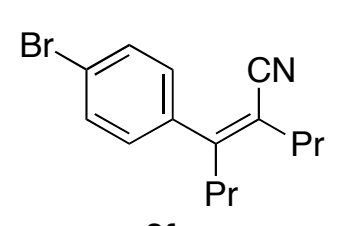

$3 f a$

(Z)-3-(4-Bromophenyl)-2-propyl-2-hexenenitrile (3fa). A colorless oil, $\mathrm{R}_{\mathrm{f}} 0.53$ (hexane-ethyl acetate $=10: 1) .{ }^{1} \mathrm{H} \mathrm{NMR}\left(400 \mathrm{MHz}, \mathrm{CDCl}_{3}\right) \delta 7.52(\mathrm{~d}, J=8.6 \mathrm{~Hz}$, $2 \mathrm{H}), 7.18(\mathrm{~d}, J=8.6 \mathrm{~Hz}, 2 \mathrm{H}), 2.48(\mathrm{t}, J=7.7 \mathrm{~Hz}, 2 \mathrm{H}), 2.35(\mathrm{t}, J=7.6 \mathrm{~Hz}, 2 \mathrm{H})$, 1.67 (sext, $J=7.5 \mathrm{~Hz}, 2 \mathrm{H}), 1.30$ (sext, $J=7.5 \mathrm{~Hz}, 2 \mathrm{H}), 1.01(\mathrm{t}, J=7.4 \mathrm{~Hz}, 3 \mathrm{H}$ ), $0.87(\mathrm{t}, J=7.3 \mathrm{~Hz}, 3 \mathrm{H}) ;{ }^{13} \mathrm{C} \mathrm{NMR}\left(101 \mathrm{MHz}, \mathrm{CDCl}_{3}\right) \delta 157.5,138.9,131.7,129.4,122.8,119.3,112.3$, 35.5, 32.4, 21.7, 21.0, 13.8, 13.5; IR (neat): 2963, 2932, 2872, 2210, 1587, 1487, 1458,1393, 1381, 1101, 1072, 1011, 831, $785 \mathrm{~cm}^{-1}$; HRMS (EI) Calcd for $\mathrm{C}_{15} \mathrm{H}_{18} \mathrm{BrN}: \mathrm{M}^{+}$, 291.0622. Found: $\mathrm{m} / \mathrm{z}$ 291.0628.

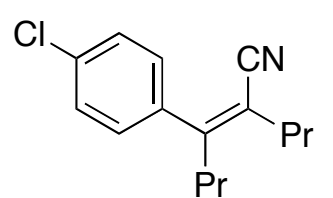

$3 g a$

(Z)-3-(4-Chlorophenyl)-2-propyl-2-hexenenitrile (3ga). A colorless oil, $\mathrm{R}_{\mathrm{f}} 0.48$ (hexane-ethyl acetate $=5: 1) .{ }^{1} \mathrm{H} \mathrm{NMR}\left(400 \mathrm{MHz}, \mathrm{CDCl}_{3}\right) \delta 7.36(\mathrm{~d}, J=8.6 \mathrm{~Hz}$, $2 \mathrm{H}), 7.24(\mathrm{~d}, J=8.6 \mathrm{~Hz}, 2 \mathrm{H}), 2.49(\mathrm{t}, J=7.7 \mathrm{~Hz}, 2 \mathrm{H}), 2.35(\mathrm{t}, J=7.6 \mathrm{~Hz}, 2 \mathrm{H})$, 1.67 (sext, $J=7.5 \mathrm{~Hz}, 2 \mathrm{H}), 1.30(\mathrm{sext}, J=7.5 \mathrm{~Hz}, 2 \mathrm{H}), 1.02(\mathrm{t}, J=7.4 \mathrm{~Hz}, 3 \mathrm{H})$, $0.87(\mathrm{t}, J=7.3 \mathrm{~Hz}, 3 \mathrm{H}) ;{ }^{13} \mathrm{C} \mathrm{NMR}\left(101 \mathrm{MHz}, \mathrm{CDCl}_{3}\right) \delta 157.5,138.5,134.6,129.2,128.7,119.3,112.3$, 35.6, 32.4, 21.7, 21.0, 13.8, 13.5; IR (neat): 2963, 2932, 2874, 2210, 1593, 1491, 1458, 1092, 1015, $835 \mathrm{~cm}^{-1}$; Anal. Calcd for $\mathrm{C}_{15} \mathrm{H}_{18} \mathrm{ClN}$; C, 72.71; H, 7.32. Found: C, 72.97; H, 7.59.

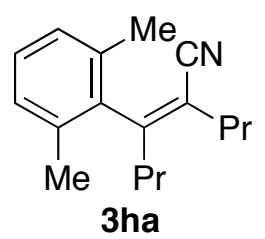

(Z)-3-(2,6-Dimethylphenyl)-2-propyl-2-hexenenitrile (3ha). A colorless oil, $\mathrm{R}_{\mathrm{f}} 0.53$ (hexane-ethyl acetate $=5: 1) .{ }^{1} \mathrm{H} \mathrm{NMR}\left(400 \mathrm{MHz}, \mathrm{CDCl}_{3}\right) \delta 7.13(\mathrm{dd}, J=8.5,6.5 \mathrm{~Hz}$, 1H), $7.06(\mathrm{~d}, J=7.3 \mathrm{~Hz}, 2 \mathrm{H}), 2.45-2.35(\mathrm{~m}, 4 \mathrm{H}), 2.22$ (s, 6H), 1.70 (sext, $J=7.4 \mathrm{~Hz}$, $2 \mathrm{H}), 1.42-1.29(\mathrm{~m}, 2 \mathrm{H}), 1.05(\mathrm{t}, J=7.3 \mathrm{~Hz}, 3 \mathrm{H}), 0.92(\mathrm{t}, J=7.2 \mathrm{~Hz}, 3 \mathrm{H}) ;{ }^{13} \mathrm{C} \mathrm{NMR}$ $\left(101 \mathrm{MHz} \mathrm{CDCl}_{3}\right) \delta 158.1,139.5,134.6,127.9,127.7,118.7,113.7,36.2,31.6,21.6,20.8,19.8,14.6$, 13.6; IR (neat): 2963, 2932, 2872, 2212, 1464, 1379, $772 \mathrm{~cm}^{-1}$; Anal. Calcd for $\mathrm{C}_{17} \mathrm{H}_{23} \mathrm{~N} ; \mathrm{C}, 84.59 ; \mathrm{H}$, 9.60. Found: C, 84.38; H, 9.71.

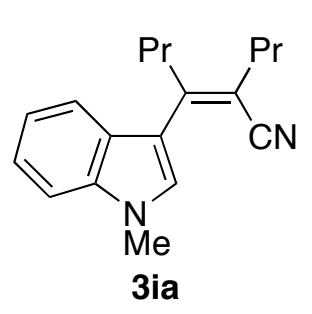

(Z)-3-(1-Methyl-3-indolyl)-2-propyl-2-hexenenitrile (3ia). A pale yellow solid, $\mathrm{mp}=74.7-75.3{ }^{\circ} \mathrm{C}, \mathrm{R}_{\mathrm{f}} 0.30$ (hexane-ethyl acetate $\left.=5: 1\right) .{ }^{1} \mathrm{H}$ NMR $(400 \mathrm{MHz}$, $\left.\mathrm{CDCl}_{3}\right) \delta 7.58(\mathrm{~d}, J=7.9 \mathrm{~Hz}, 1 \mathrm{H}), 7.34(\mathrm{~d}, J=8.1 \mathrm{~Hz}, 1 \mathrm{H}), 7.30-7.23(\mathrm{~m}, 2 \mathrm{H})$, $7.17(\mathrm{t}, J=7.5 \mathrm{~Hz}, 1 \mathrm{H}), 3.81(\mathrm{~s}, 3 \mathrm{H}), 2.66(\mathrm{t}, J=7.8 \mathrm{~Hz}, 2 \mathrm{H}), 2.41(\mathrm{t}, J=7.6 \mathrm{~Hz}$, 2H), 1.71 (sext, $J=7.5 \mathrm{~Hz}, 2 \mathrm{H}), 1.36$ (sext, $J=7.6 \mathrm{~Hz}, 2 \mathrm{H}), 1.05$ (t, $J=7.4 \mathrm{~Hz}$, $3 \mathrm{H}), 0.87(\mathrm{t}, J=7.4 \mathrm{~Hz}, 3 \mathrm{H}) ;{ }^{13} \mathrm{C} \mathrm{NMR}\left(101 \mathrm{MHz}, \mathrm{CDCl}_{3}\right) \delta 152.4,137.0,129.0,126.3,122.0,120.9$, 120.2, 119.9, 114.3, 109.7, 109.4, 35.3, 33.0, 32.4, 22.0, 21.8, 13.9, 13.6; IR (KBr): 2961, 2870, 2201, 1614, 1605, 1537, 1477, 1466, 1385, 1331, 1244, 1134, 1105, 1090, 1015, 845, $741 \mathrm{~cm}^{-1}$; Anal. Calcd 
for $\mathrm{C}_{18} \mathrm{H}_{22} \mathrm{~N}_{2} ; \mathrm{C}, 81.16 ; \mathrm{H}, 8.32$. Found: $\mathrm{C}, 81.02 ; \mathrm{H}, 8.47$.

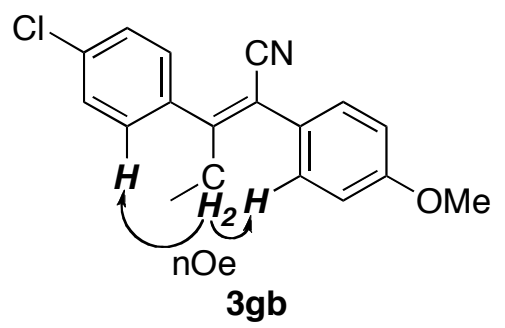

(Z)-3-(4-Chlorophenyl)-2-(4-methoxyhenyl)-2-pentenenitrile (3gb). A colorless solid, $\mathrm{mp}=109.8-110.5^{\circ} \mathrm{C}, \mathrm{R}_{\mathrm{f}} 0.34$ (hexane-ethyl acetate $=10: 1) .{ }^{1} \mathrm{H}$ NMR $\left(400 \mathrm{MHz}, \mathrm{CDCl}_{3}\right) \delta 7.46-7.30(\mathrm{~m}, 6 \mathrm{H}), 6.96(\mathrm{~d}, J=$ $8.8 \mathrm{~Hz}, 2 \mathrm{H}), 3.85(\mathrm{~s}, 3 \mathrm{H}), 2.58(\mathrm{q}, J=7.5 \mathrm{~Hz}, 2 \mathrm{H}), 0.93(\mathrm{t}, J=7.5 \mathrm{~Hz}$, $3 \mathrm{H}) ;{ }^{13} \mathrm{C}$ NMR $\left(101 \mathrm{MHz}, \mathrm{CDCl}_{3}\right) \delta 159.9,159.8,137.5,135.0,130.1$, 129.3, 128.9, 126.4, 119.2, 114.2, 112.1, 55.4, 27.5, 12.8; IR (KBr): 2980, 2963, 2934, 2841, 2206, $1605,1589,1570,1510,1491,1464,1445,1302,1283,1254,1177,1105,1084,1036,1011,845,829$, $689,515 \mathrm{~cm}^{-1}$; Anal. Calcd for $\mathrm{C}_{18} \mathrm{H}_{16} \mathrm{ClNO}$; C, 72.60; H, 5.42. Found: C, 72.68; H, 5.67.

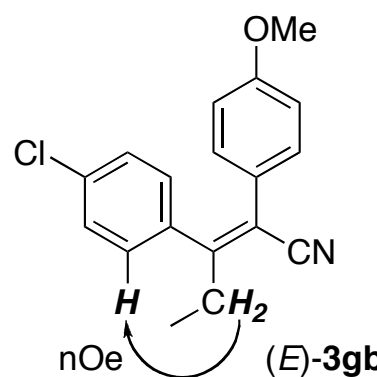

(E)-3-(4-Chlorophenyl)-2-(4-methoxyhenyl)-2-pentenenitrile [(E)-3gb]. A pale yellow oil, $\mathrm{R}_{\mathrm{f}} 0.26$ (hexane-ethyl acetate $\left.=10: 1\right) .{ }^{1} \mathrm{H}$ NMR $(400 \mathrm{MHz}$, $\left.\mathrm{CDCl}_{3}\right) \delta 7.22(\mathrm{~d}, J=8.4 \mathrm{~Hz}, 2 \mathrm{H}), 7.03-6.97(\mathrm{~m}, 4 \mathrm{H}), 6.70(\mathrm{~d}, J=8.8 \mathrm{~Hz}, 2 \mathrm{H})$, $3.76(\mathrm{~s}, 3 \mathrm{H}), 2.92(\mathrm{q}, J=7.5 \mathrm{~Hz}, 2 \mathrm{H}), 1.06(\mathrm{t}, J=7.5 \mathrm{~Hz}, 3 \mathrm{H}) ;{ }^{13} \mathrm{C}$ NMR $(101$ $\left.\mathrm{MHz}, \mathrm{CDCl}_{3}\right) \delta 159.3,158.2,136.4,134.3,130.7,129.9,128.8,125.7,118.8$, 1464, 1294, 1254, 1178, 1092, 1034, 1015, 912, 826, $733 \mathrm{~cm}^{-1}$; HRMS (EI) Calcd for $\mathrm{C}_{18} \mathrm{H}_{16}$ ClNO: ${ }^{+}$, 297.0920. Found: $m / z 297.0932$.

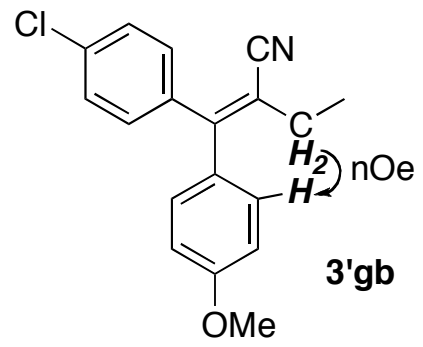

(Z)-3-(4-Chlorophenyl)-3-(4-methoxyhenyl)-2-ethylacrylonitrile (3'gb). A pale yellow oil, $\mathrm{R}_{\mathrm{f}} 0.26$ (hexane-ethyl acetate $\left.=10: 1\right) .{ }^{1} \mathrm{H}$ NMR $(400$ $\left.\mathrm{MHz}, \mathrm{CDCl}_{3}\right) \delta 7.33$ (d, $\left.J=8.4 \mathrm{~Hz}, 2 \mathrm{H}\right), 7.27$ (d, $\left.J=8.4 \mathrm{~Hz}, 2 \mathrm{H}\right), 7.03$ (d, $J$ $=9.0 \mathrm{~Hz}, 2 \mathrm{H}), 6.90(\mathrm{~d}, J=8.4 \mathrm{~Hz}, 2 \mathrm{H}), 3.83(\mathrm{~s}, 3 \mathrm{H}), 2.42(\mathrm{q}, J=7.5 \mathrm{~Hz}$, $2 \mathrm{H}), 1.24(\mathrm{t}, J=7.4 \mathrm{~Hz}, 3 \mathrm{H}) ;{ }^{13} \mathrm{C} \mathrm{NMR}\left(101 \mathrm{MHz}, \mathrm{CDCl}_{3}\right) \delta 160.1,154.9$, 138.7, 135.2, 130.9, 130.8, 130.6, 128.5, 119.7, 113.8, 113.0, 55.3, 25.8, 13.3; IR (neat): 2974, 2206, $1607,1510,1489,1460,1288,1252,1175,1092,1032,1015,908,833,824,731 \mathrm{~cm}^{-1}$; Calcd for $\mathrm{C}_{18} \mathrm{H}_{16} \mathrm{ClNO}$; , 72.60; H, 5.42. Found: C, 72.40; H, 5.24.

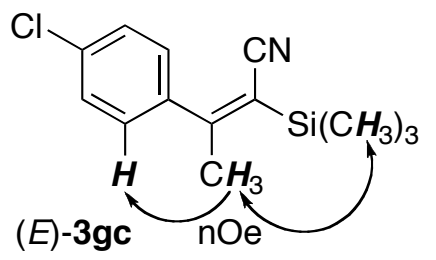

(E)-3-(4-Chlorophenyl)-2-trimethylsilyl-2-butenenitrile (3gc). A colorless oil, $\mathrm{R}_{\mathrm{f}} 0.14$ (hexane-ethyl acetate $\left.=30: 1\right) .{ }^{1} \mathrm{H}$ NMR $(400 \mathrm{MHz}$, $\left.\mathrm{CDCl}_{3}\right) \delta$ 7.39-7.33 (m, 4H), 2.31, (s, 3H), $0.39(\mathrm{~s}, 9 \mathrm{H}) ;{ }^{13} \mathrm{C}$ NMR $(101$ $\left.\mathrm{MHz}, \mathrm{CDCl}_{3}\right) \delta 168.1,140.5,134.9,128.6,128.1,120.1,111.2,24.5,-0.2$; 
IR (neat): 2959, 2195, 1595, 1578, 1556, 1489, 1254, 1103, 1013, 845, 760, $673 \mathrm{~cm}^{-1}$; Anal. Calcd [as a mixture with (Z)-3gc and 3'gc] for $\mathrm{C}_{13} \mathrm{H}_{16} \mathrm{ClNSi}$; C, 62.50; H, 6.46. Found: C, 62.75; H, 6.52.

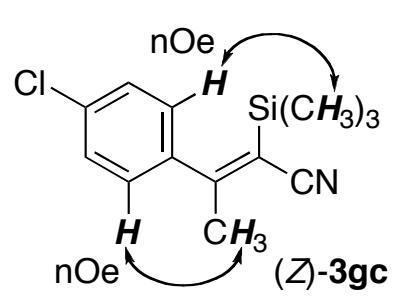

(Z)-3-(4-Chlorophenyl)-2-trimethylsilyl-2-butenenitrile $\quad[(Z)-3 g c] . \quad A$ colorless oil, $\mathrm{R}_{\mathrm{f}} 0.14$ (hexane-ethyl acetate $\left.=30: 1\right) .{ }^{1} \mathrm{H}$ NMR $(400 \mathrm{MHz}$, $\left.\mathrm{CDCl}_{3}\right) \delta 7.35(\mathrm{~d}, J=8.6 \mathrm{~Hz}, 2 \mathrm{H}), 7.09(\mathrm{~d}, J=8.6 \mathrm{~Hz}, 2 \mathrm{H}), 2.46(\mathrm{~s}, 3 \mathrm{H})$, $-0.01(\mathrm{~s}, 9 \mathrm{H}) ;{ }^{13} \mathrm{C} \mathrm{NMR}\left(101 \mathrm{MHz}, \mathrm{CDCl}_{3}\right) \delta 169.7,140.3,134.7,128.5$, 128.1, 119.7, 113.0, 28.2, -0.2; IR (neat): 2959, 2899, 2197, 1599, 1576, 1485, $1435,1254,1105,1090,1015,982,845,762,698,633,554 \mathrm{~cm}^{-1}$.

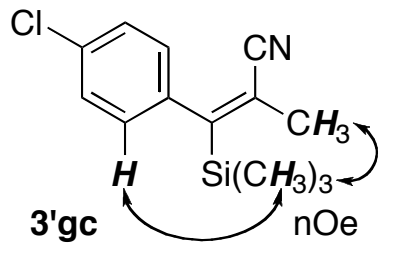

(E)-3-(4-Chlorophenyl)-3-trimethylsilyl-2-methylpropenenitrile (3'gc). A colorless solid, $\mathrm{mp}=67.2-67.9^{\circ} \mathrm{C}, \mathrm{R}_{\mathrm{f}} 0.14$ (hexane-ethyl acetate $=30: 1$ ). ${ }^{1} \mathrm{H}$ NMR (400 MHz, $\left.\mathrm{CDCl}_{3}\right) \delta 7.33(\mathrm{~d}, J=8.5 \mathrm{~Hz}, 2 \mathrm{H}), 6.92(\mathrm{~d}, J=8.5 \mathrm{~Hz}, 2 \mathrm{H})$, 2.19 (s, 3H), 0.16 (s, 9H); $\left.{ }^{13} \mathrm{C} \mathrm{NMR} \mathrm{(101} \mathrm{MHz,} \mathrm{CDCl}_{3}\right) \delta$ 161.9, 140.9, 132.9, 128.6, 127.7, 119.9, 118.3, 20.5, -0.2; IR (KBr): 2957, 2214, 1580, 1487, 1250, 1088, 1013, 908, 843, $800,760,521 \mathrm{~cm}^{-1}$.

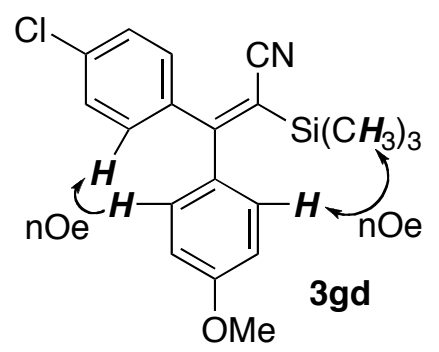

\section{(E)-3-(4-Chlorophenyl)-3-(4-methoxyphenyl)-2-trimethylsilylacrylonitri}

le (3gd). A colorless oil, $\mathrm{R}_{\mathrm{f}} 0.38$ (hexane-ethyl acetate $\left.=5: 1\right)$. ${ }^{1} \mathrm{H}$ NMR (400 $\left.\mathrm{MHz}, \mathrm{CDCl}_{3}\right) \delta 7.35-7.29(\mathrm{~m}, 4 \mathrm{H}), 7.07(\mathrm{~d}, J=8.8 \mathrm{~Hz}, 2 \mathrm{H}), 6.88(\mathrm{~d}, J=8.8$ $\mathrm{Hz}, 2 \mathrm{H}), 3.85$ (s, 3H), 0.10 (s, 9H); $\left.{ }^{13} \mathrm{C} \mathrm{NMR} \mathrm{(101} \mathrm{MHz,} \mathrm{CDCl}_{3}\right) \delta 170.0$, $160.6,139.6,135.7,133.2,130.8,130.7,128.3,121.0,113.5,111.3,55.4$, 0.0; IR (neat): 2957, 2899, 2839, 2189, 1607, 1508, 1487, 1304, 1288, 1252, 1175, 1092, 1032, 1015, 845, 802, $760 \mathrm{~cm}^{-1}$; Anal. Calcd for $\mathrm{C}_{19} \mathrm{H}_{20}$ ClNOSi; C, 66.74; H, 5.90. Found: C, 66.92; H, 5.86.

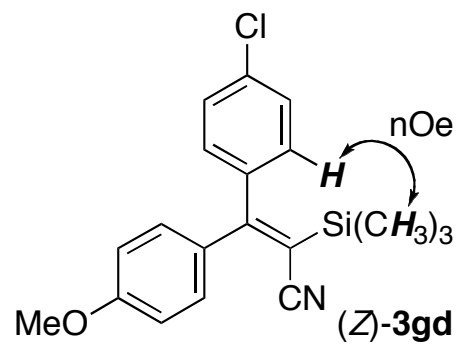

(Z)-3-(4-Chlorophenyl)-3-(4-methoxyphenyl)-2-trimethylsilylacrylonit rile $[(Z)-3 g d]$. A colorless solid, $\mathrm{mp}=98.7-99.6{ }^{\circ} \mathrm{C}, \quad \mathrm{R}_{\mathrm{f}} \quad 0.35$ (hexane-ethyl acetate $=5: 1) .{ }^{1} \mathrm{H}$ NMR $\left(400 \mathrm{MHz}, \mathrm{CDCl}_{3}\right) \delta 7.36(\mathrm{~d}, J=$ $8.5 \mathrm{~Hz}, 2 \mathrm{H}), 7.33(\mathrm{~d}, J=8.8 \mathrm{~Hz}, 2 \mathrm{H}), 7.10(\mathrm{~d}, J=8.5 \mathrm{~Hz}, 2 \mathrm{H}), 6.87(\mathrm{~d}, J$ 169.4, 160.8, 139.8, 135.3, 132.9, 131.0, 130.7, 128.3, 121.1, 113.5, 110.1, 55.4, -0.01; IR (KBr): 2961, 2191, 1601, 1572, 1543, 1508, 1489, 1306, 1252, 1182, 1167, 1092, 1028, 860, $837 \mathrm{~cm}^{-1}$; Anal. Calcd for $\mathrm{C}_{19} \mathrm{H}_{20} \mathrm{ClNOSi}$;, $66.74 ; \mathrm{H}, 5.90$. Found: C, 66.48; H, 5.97. 
Nickel-LA-catalyzed arylcyanation of alkynes using dichlorobis(dimethylphenyphosphine)nickel(II) as a precatalyst. A solution of $\left(\mathrm{PhMe}_{2} \mathrm{P}\right)_{2} \mathrm{NiCl}_{2}(4.1 \mathrm{mg}, 0.010 \mathrm{mmol})$ in toluene $(1.0 \mathrm{~mL}), 2 \mathrm{a}(110 \mathrm{mg}, 1.00 \mathrm{mmol})$, a $1.0 \mathrm{M}$ solution of $\mathrm{AlMe}_{3}$ in hexane $(0.040 \mathrm{~mL}, 0.040 \mathrm{mmol})$, and $\mathrm{C}_{12} \mathrm{H}_{26}$ (internal standard, $\left.56 \mathrm{mg}, 0.33 \mathrm{mmol}\right)$ were added to $1 \mathrm{a}(133 \mathrm{mg}, 1.00 \mathrm{mmol})$ in a dry box. The vial was taken outside the dry box and heated at $50{ }^{\circ} \mathrm{C}$ for $19 \mathrm{~h}$. The resulting mixture was filtered through a silica gel pad, concentrated in vacuo, and purified by flash silica gel column chromatography (hexane-ethyl acetate $=8: 1)$ to give 3aa $(233 \mathrm{mg}, 96 \%)$.

Nickel-LA-catalyzed alkenylcyanation of alkynes. A general procedure. An alkenyl cyanide (1.00 mmol), $\mathrm{BPh}_{3}(19.4 \mathrm{mg}, 0.080 \mathrm{mmol})$, an alkyne $(1.2 \mathrm{mmol})$, and $\mathrm{C}_{12} \mathrm{H}_{26}$ (internal standard, $85 \mathrm{mg}, 0.50 \mathrm{mmol})$ were added to a solution of $\mathrm{Ni}(\mathrm{cod})_{2}(5.5 \mathrm{mg}, 0.020 \mathrm{mmol})$ and $\mathrm{PMe}_{3}(3.0 \mathrm{mg}$, $0.040 \mathrm{mmol})$ in toluene $(1.0 \mathrm{~mL})$ in a dry box. The vial was taken outside the dry box and heated at $80{ }^{\circ} \mathrm{C}$ for the time specified Table 2. The resulting mixture was filtered through a silica gel pad, concentrated in vacuo, and purified by flash silica gel column chromatography to give the corresponding alkenylcyanation products in yields listed in Table 2.

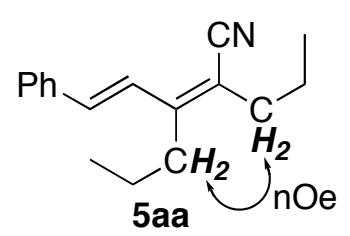

(2Z,4E)-5-Phenyl-2,3-dipropyl-2,4-pentadienenitrile (5aa). A pale yellow oil, $\mathrm{R}_{\mathrm{f}} 0.13$ (hexane-ethyl acetate $\left.=30: 1\right) .{ }^{1} \mathrm{H} \mathrm{NMR}\left(400 \mathrm{MHz}, \mathrm{CDCl}_{3}\right) \delta 7.52(\mathrm{~d}, J=$ $7.1 \mathrm{~Hz}, 2 \mathrm{H}), 7.40-7.26(\mathrm{~m}, 4 \mathrm{H}), 6.84(\mathrm{~d}, J=16.1 \mathrm{~Hz}, 1 \mathrm{H}), 2.46(\mathrm{t}, J=8.0 \mathrm{~Hz}$, $2 \mathrm{H}), 2.33(\mathrm{t}, J=7.6 \mathrm{~Hz}, 2 \mathrm{H}), 1.66$ (sext, $J=7.5 \mathrm{~Hz}, 2 \mathrm{H}), 1.53$ (sext, $J=7.6 \mathrm{~Hz}$, 2H) $1.02(\mathrm{t}, J=7.5 \mathrm{~Hz}, 3 \mathrm{H}), 1.00(\mathrm{t}, J=7.4 \mathrm{~Hz}, 3 \mathrm{H}) ;{ }^{13} \mathrm{C} \mathrm{NMR}\left(101 \mathrm{MHz}, \mathrm{CDCl}_{3}\right) \delta 153.1,136.2$, 133.5, 128.8, 128.7, 127.1, 127.0, 119.1, 112.4, 32.2, 29.9, 22.6, 21.8, 14.3, 13.6; IR (neat): 2963, 2934, 2874, 2203, 1692, 1450, 962, 754, $692 \mathrm{~cm}^{-1}$; Anal. Calcd for $\mathrm{C}_{17} \mathrm{H}_{21} \mathrm{~N}$; C, 85.30; H, 8.84. Found: C, $85.54 ; \mathrm{H}, 8.78$.

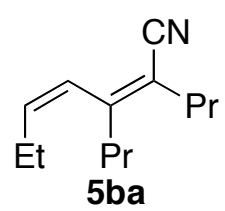

(2Z,4Z)-2,3-dipropyl-2,4-heptadienenitrile [(Z)-5ba]. A colorless oil, $\mathrm{R}_{\mathrm{f}} 0.15$ (hexane-ethyl acetate $=30: 1) .{ }^{1} \mathrm{H} \mathrm{NMR}\left(400 \mathrm{MHz}, \mathrm{CDCl}_{3}\right) \delta 5.89(\mathrm{~d}, J=11.7 \mathrm{~Hz}, 1 \mathrm{H})$, $5.66(\mathrm{dt}, J=11.7,7.3 \mathrm{~Hz}, 1 \mathrm{H}), 2.25(\mathrm{t}, J=7.6 \mathrm{~Hz}, 2 \mathrm{H}), 2.20(\mathrm{t}, J=7.7 \mathrm{~Hz}, 2 \mathrm{H}), 2.13$ (qdd, $J=7.5,7.3,1.8 \mathrm{~Hz}, 2 \mathrm{H}), 1.62(\mathrm{sext}, J=7.4 \mathrm{~Hz}, 2 \mathrm{H}), 1.42(\mathrm{sext}, J=7.5 \mathrm{~Hz}, 2 \mathrm{H})$, $1.03(\mathrm{t}, J=7.5 \mathrm{~Hz}, 3 \mathrm{H}), 0.98(\mathrm{t}, J=7.3 \mathrm{~Hz}, 3 \mathrm{H}), 0.92(\mathrm{t}, J=7.3 \mathrm{~Hz}, 3 \mathrm{H}) ;{ }^{13} \mathrm{C} \mathrm{NMR}(101 \mathrm{MHz}$, $\left.\mathrm{CDCl}_{3}\right) \delta 154.7,137.6,126.8,119.5,111.5,34.4,31.8,22.9,21.9,21.3,14.1,13.9,13.7$; IR (neat): 2964, 2934, 2874, 2208, 1458, $1379 \mathrm{~cm}^{-1}$; HRMS (EI) Calcd for $\mathrm{C}_{13} \mathrm{H}_{21} \mathrm{~N}: \mathrm{M}^{+}, 191.1674$. Found: $\mathrm{m} / z$ 191.1677. 
<smiles>C/C=C/C(CC)=C(/C#N)CC</smiles>

(E)-5ba

(2Z,4E)-2,3-dipropyl-2,4-heptadienenitrile [(E)-5ba]. A colorless oil, $\mathrm{R}_{\mathrm{f}} 0.15$ (hexane-ethyl acetate $=30: 1) .{ }^{1} \mathrm{H} \mathrm{NMR}\left(400 \mathrm{MHz}, \mathrm{CDCl}_{3}\right) \delta 6.60(\mathrm{dt}, J=15.6,1.5$ $\mathrm{Hz}, 1 \mathrm{H}), 6.07$ (dt, $J=15.6,6.7 \mathrm{~Hz}, 1 \mathrm{H}), 2.32(\mathrm{t}, J=8.0 \mathrm{~Hz}, 2 \mathrm{H}), 2.28-2.19(\mathrm{~m}, 4 \mathrm{H})$, $1.61(\operatorname{sext}, J=7.5 \mathrm{~Hz}, 2 \mathrm{H}), 1.44(\mathrm{sext}, J=7.6 \mathrm{~Hz}, 2 \mathrm{H}), 1.07(\mathrm{t}, J=7.4 \mathrm{~Hz}, 3 \mathrm{H})$, $0.972(\mathrm{t}, J=7.3 \mathrm{~Hz}, 3 \mathrm{H}), 0.966(\mathrm{t}, J=7.4 \mathrm{~Hz}, 3 \mathrm{H}) ;{ }^{13} \mathrm{C} \mathrm{NMR}\left(101 \mathrm{MHz}, \mathrm{CDCl}_{3}\right) \delta$ 153.4, 138.3, 128.0, 119.2, 109.8, 32.0, 30.1, 26.4, 22.7, 21.9, 14.4, 13.8, 13.5; IR (neat): 2964, 2934, 2874, 2205, 1638, 1570, 1462, 1381, 1088, $966 \mathrm{~cm}^{-1}$; HRMS (EI) Calcd for $\mathrm{C}_{13} \mathrm{H}_{21} \mathrm{~N}: \mathrm{M}^{+}, 191.1674$. Found: $m / z$ 191.1675.

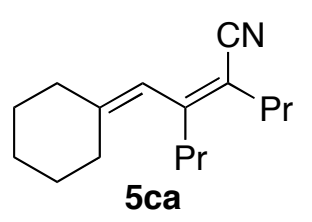

(Z)-2,3-Dipropyl-4-cyclohexylidene-2-butenenitrile (5ca). A pale yellow oil, $\mathrm{R}_{\mathrm{f}}$ 0.28 (hexane-ethyl acetate $=40: 1) .{ }^{1} \mathrm{H} \mathrm{NMR}\left(400 \mathrm{MHz}, \mathrm{CDCl}_{3}\right) \delta 5.62(\mathrm{~s}, 1 \mathrm{H})$, 2.27-2.10 (m, 8H), 1.65-1.52 (m, 8H), 1.40 (sext, $J=7.5 \mathrm{~Hz}, 2 \mathrm{H}), 0.96(\mathrm{t}, J=7.4$ $\mathrm{Hz}, 3 \mathrm{H}), 0.90(\mathrm{t}, J=7.4 \mathrm{~Hz}, 3 \mathrm{H}) ;{ }^{13} \mathrm{C} \mathrm{NMR}\left(101 \mathrm{MHz}, \mathrm{CDCl}_{3}\right) \delta 155.3,146.6$, 121.0, 119.9, 111.1, 37.1, 34.7, 31.7, 30.6, 28.3, 27.1, 26.4, 21.8, 21.1, 14.0, 13.5; IR (neat): 2961, 2932, 2872, 2856, 2208, 1647, 1611, 1448, 1379, 1342, 1234, 1109, 1088, 833, $735 \mathrm{~cm}^{-1}$; Anal. Calcd for $\mathrm{C}_{16} \mathrm{H}_{25} \mathrm{~N}$; C, 83.06; H, 10.89. Found: C, 82.85; H, 10.71 .

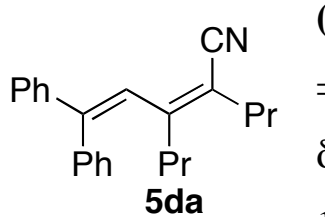

(Z)-5,5-Diphenyl-2,3-dipropyl-2,4-pentadienenitrile (5da). A colorless solid, mp $=58.1-58.7^{\circ} \mathrm{C}, \mathrm{R}_{\mathrm{f}} 0.25$ (hexane-ethyl acetate $\left.=20: 1\right) .{ }^{1} \mathrm{H}$ NMR $\left(400 \mathrm{MHz}, \mathrm{CDCl}_{3}\right)$ $\delta 7.40-7.16(\mathrm{~m}, 10 \mathrm{H}), 6.83(\mathrm{~s}, 1 \mathrm{H}), 2.20(\mathrm{t}, J=7.5 \mathrm{~Hz}, 2 \mathrm{H}), 1.89$ (t, $J=7.8 \mathrm{~Hz}, 2 \mathrm{H})$, 1.54 (sext, $J=7.5 \mathrm{~Hz}, 2 \mathrm{H}), 1.32$ (sext, $J=7.5 \mathrm{~Hz}, 2 \mathrm{H}), 0.91$ (t, $J=7.3 \mathrm{~Hz}, 3 \mathrm{H}), 0.74$ (t, $J=7.4 \mathrm{~Hz}, 3 \mathrm{H}) ;{ }^{13} \mathrm{C} \mathrm{NMR}\left(101 \mathrm{MHz}, \mathrm{CDCl}_{3}\right) \delta 155.6,146.8,142.2,139.8,129.9,128.2,128.0$, 126.6, 119.5, 113.6, 32.8, 31.8, 22.0, 21.7, 13.9, 13.5; IR (KBr): 2963, 2932, 2870, 2203, 1599, 1493, 1445, 1375, 870, 779, 762, $696 \mathrm{~cm}^{-1}$; Anal. Calcd for $\mathrm{C}_{23} \mathrm{H}_{25} \mathrm{~N}$; C, 87.57; H, 7.99. Found: C, 87.46; H, 8.04 .

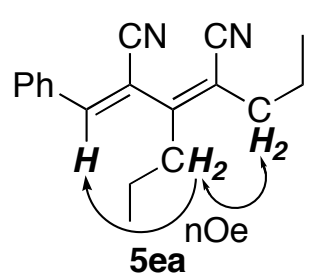

(2Z,4Z)-4-Cyano-5-phenyl-2,3-dipropyl-2,4-pentadienenitrile (5ea). A pale yellow oil, $\mathrm{R}_{\mathrm{f}} 0.10$ (hexane-ethyl acetate $\left.=20: 1\right) .{ }^{1} \mathrm{H} \mathrm{NMR}\left(400 \mathrm{MHz}, \mathrm{CDCl}_{3}\right) \delta$ 7.89-7.81 (m, 2H), 7.49-7.44 (m, 3H), $7.36(\mathrm{~s}, 1 \mathrm{H}), 2.49(\mathrm{t}, J=7.8 \mathrm{~Hz}, 2 \mathrm{H}), 2.36$ (t, $J=7.7 \mathrm{~Hz}, 2 \mathrm{H}), 1.69$ (sext, $J=7.5 \mathrm{~Hz}, 2 \mathrm{H}), 1.53$ (sext, $J=7.5 \mathrm{~Hz}, 2 \mathrm{H}), 1.03$ (t, $J=7.3 \mathrm{~Hz}, 3 \mathrm{H}), 0.98(\mathrm{t}, J=7.4 \mathrm{~Hz}, 3 \mathrm{H}) ;{ }^{13} \mathrm{C} \mathrm{NMR}\left(101 \mathrm{MHz}, \mathrm{CDCl}_{3}\right) \delta 152.0$, 148.2 , 132.4, 131.3, 129.4, 128.9, 118.1, 116.3, 114.7, 109.6, 33.0, 32.8, 21.7, 21.4, 13.9, 13.7; IR (neat): 2964, 2933, 2874, 2212, 1605, 1574, 1448, 1381, 1092, 935, 758, $691 \mathrm{~cm}^{-1}$; Anal. Calcd for 
$\mathrm{C}_{18} \mathrm{H}_{20} \mathrm{~N}_{2} ; \mathrm{C}, 81.78 ; \mathrm{H}, 7.63$. Found: C, 81.83; H, 7.66.

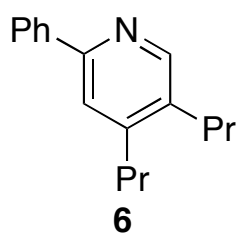

Conversion of 5aa to 3,4-dipropyl-1-phenylpyridine (6). To a solution of 5aa (72 $\mathrm{mg}, 0.30 \mathrm{mmol})$ in toluene $(15 \mathrm{~mL})$ was added a $1.5 \mathrm{M}$ solution of DIBAL-H in toluene $(0.40 \mathrm{~mL}, 0.60 \mathrm{mmol})$ at $0{ }^{\circ} \mathrm{C}$, and the resulting mixture was stirred at the same temperature for $15 \mathrm{~min}$. The reaction was quenched with $\mathrm{MeOH}(0.150 \mathrm{~mL})$ at $0{ }^{\circ} \mathrm{C}$ and heated at $100{ }^{\circ} \mathrm{C}$ for $5 \mathrm{~h}$ in the open air. To the resulting mixture was added a slurry of $\mathrm{SiO}_{2}(3.0 \mathrm{~g})$ in water $(0.90 \mathrm{~mL})$, and the whole was stirred at $\mathrm{rt}$ for $45 \mathrm{~min}$. Anhydrous $\mathrm{MgSO}_{4}(0.50 \mathrm{~g})$ and $\mathrm{K}_{2} \mathrm{CO}_{3}$ $(0.50 \mathrm{~g})$ were added, and the resulting mixture was further stirred for $90 \mathrm{~min}$, filtered through a Celite pad, and concentrated in vacuo. The residue was purified by flash chromatography on silica gel (hexane-ethyl acetate $=35: 1)$ to give $6(44 \mathrm{mg}, 61 \%)$ as a pale yellow oil, $\mathrm{R}_{\mathrm{f}} 0.43$ (hexane-ethyl acetate = 7:1). ${ }^{1} \mathrm{H}$ NMR $\left(400 \mathrm{MHz}, \mathrm{CDCl}_{3}\right) \delta 8.43(\mathrm{~s}, 1 \mathrm{H}), 7.99-7.93(\mathrm{~m}, 2 \mathrm{H}), 7.50(\mathrm{~s}, 1 \mathrm{H}), 7.48-7.42$ (m, 2H), 7.41-7.34 (m, 1H), 2.68-2.59 (m, 4H), 1.74-1.58 (m, 4H), 1.02 (t, $J=7.3 \mathrm{~Hz}, 3 \mathrm{H}), 1.01$ (t, $J$ $=7.2 \mathrm{~Hz}, 3 \mathrm{H}) ;{ }^{13} \mathrm{C} \mathrm{NMR}\left(101 \mathrm{MHz}, \mathrm{CDCl}_{3}\right) \delta$ 155.0, 150.3, 149.8, 139.6, 134.5, 128.6, 128.4, 126.7, 120.7, 34.1, 31.8, 24.1, 23.5, 14.11, 14.07; IR (neat): 2959, 2932, 2870, 1597, 1477, 1377, 777, 694 $\mathrm{cm}^{-1}$; Anal. Calcd for $\mathrm{C}_{17} \mathrm{H}_{21} \mathrm{~N} ; \mathrm{C}, 85.30 ; \mathrm{H}, 8.84$. Found: $\mathrm{C}, 85.51 ; \mathrm{H}, 9.12$.

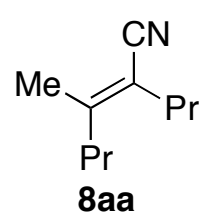

Nickel-LA-catalyzed methylcyanation of 4-octyne (2a) with a $10 \mathrm{mmol}$ scale. Acetonitrile $(7 \mathbf{a}, 0.41 \mathrm{~g}, 10.0 \mathrm{mmol})$, a $1.0 \mathrm{M}$ solution of $\mathrm{AlMe}_{3}$ in hexane $(0.20 \mathrm{~mL}, 2.0$ $\mathrm{mmol})$, and $2 \mathrm{a}(1.10 \mathrm{~g}, 10.0 \mathrm{mmol})$ were added sequentially to a solution of $\mathrm{Ni}(\mathrm{cod})_{2}$ $(138 \mathrm{mg}, 0.50 \mathrm{mmol})$ and $\mathrm{PPh}_{2}(t-\mathrm{Bu})(0.24 \mathrm{~g}, 1.00 \mathrm{mmol})$ in toluene $(10 \mathrm{~mL})$ in a dry box. After being stirred at $80{ }^{\circ} \mathrm{C}$ for $4 \mathrm{~h}$, the resulting mixture was filtered through a silica gel pad, and concentrated in vacuo. The residue was distilled to give (E)-3-methyl-2-propyl-2-hexenenitrile (8aa, $1.1 \mathrm{~g}, 71 \%)$ as a pale yellow oil, bp $80{ }^{\circ} \mathrm{C}(20 \mathrm{mmHg}), \mathrm{R}_{\mathrm{f}} 0.18$ (hexane-ethyl acetate $\left.=40: 1\right) .{ }^{1} \mathrm{H}$ NMR $\left(400 \mathrm{MHz}, \mathrm{CDCl}_{3}\right) \delta 2.21-2.12(\mathrm{~m}, 4 \mathrm{H}), 2.05$ (s, 3H), 1.56 (sext, $\left.J=7.5 \mathrm{~Hz}, 2 \mathrm{H}\right), 1.46$ (sext, $J=7.5$ $\mathrm{Hz}, 2 \mathrm{H}), 0.94(\mathrm{t}, J=7.3 \mathrm{~Hz}, 3 \mathrm{H}), 0.93(\mathrm{t}, J=7.3 \mathrm{~Hz}, 3 \mathrm{H}) ;{ }^{13} \mathrm{C} \mathrm{NMR}\left(101 \mathrm{MHz}, \mathrm{CDCl}_{3}\right) \delta 155.3,119.3$, 109.7, 35.6, 31.5, 22.6, 21.9, 21.0, 14.1, 13.6; IR (neat): 2964, 2934, 2874, 2208, 1630, 1466, 1381, 1138, 1094, $741 \mathrm{~cm}^{-1}$; Anal. Calcd for $\mathrm{C}_{10} \mathrm{H}_{17} \mathrm{~N} ; \mathrm{C}, 79.41 ; \mathrm{H}, 11.33$. Found: C, 79.47; H, 11.47.

Nickel-LA-catalyzed alkylcyanation of 4-octyne (2a). A general procedure. An alkyl cyanide $(1.00 \mathrm{mmol})$, a solution of an aluminum Lewis acid $(0.20 \mathrm{mmol})$ in hexane, $2 \mathrm{a}(1.00 \mathrm{mmol})$, and $\mathrm{C}_{12} \mathrm{H}_{26}$ (internal standard, $85 \mathrm{mg}, 0.50 \mathrm{mmol}$ ) were added sequentially to a solution of $\mathrm{Ni}(\operatorname{cod})_{2}(14$ $\mathrm{mg}, 0.050 \mathrm{mmol})$ and a ligand $(0.10 \mathrm{mmol})$ in toluene $(1.0 \mathrm{~mL})$ in a dry box. The vial was taken 
outside the dry box and heated at $80{ }^{\circ} \mathrm{C}$ for the time specified in Table 3 . The resulting mixture was filtered through a silica gel pad, concentrated in vacuo, and purified by flash silica gel column chromatography to give the corresponding alkylcyanation products in yields listed in Table 3 .

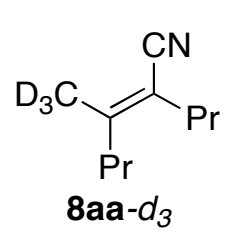

$(\boldsymbol{E})-3-\left({ }^{2} \mathbf{H}_{3}\right)$ Methyl-2-propyl-2-hexenenitrile $\quad\left(\mathbf{8 a a}-\boldsymbol{d}_{3}\right)$. A colorless oil, $\mathrm{R}_{\mathrm{f}} \quad 0.20$ (hexane-ethyl acetate $=40: 1) .{ }^{1} \mathrm{H}$ NMR $\left(400 \mathrm{MHz}, \mathrm{CDCl}_{3}\right) \delta 2.21-2.11(\mathrm{~m}, 4 \mathrm{H}), 1.55$ $(\operatorname{sext}, J=7.5 \mathrm{~Hz}, 2 \mathrm{H}), 1.45(\mathrm{sext}, J=7.5 \mathrm{~Hz}, 2 \mathrm{H}), 0.94(\mathrm{t}, J=7.3 \mathrm{~Hz}, 3 \mathrm{H}), 0.92$ (t, $J=$ $7.3 \mathrm{~Hz}, 3 \mathrm{H}) ;{ }^{13} \mathrm{C}$ NMR (101 $\left.\mathrm{MHz}, \mathrm{CDCl}_{3}\right) \delta$ 155.2, 119.3, 109.7, 35.5, 31.5, 21.9, 21.0, 14.1, 13.6; IR (neat): 2963, 2934, 2874, 2208, 1624, 1466, 1381, 1090, 1042, $739 \mathrm{~cm}^{-1}$; HRMS (EI) Calcd for $\mathrm{C}_{10} \mathrm{H}_{14} \mathrm{D}_{3} \mathrm{~N}: \mathrm{M}^{+}, 154.1549$. Found: $m / z$, 154.1557.

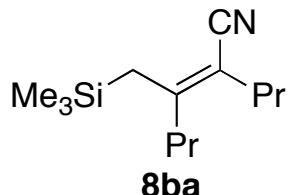

$8 \mathrm{ba}$

(Z)-3-(Trimethylsilyl)methyl-2-propyl-2-hexenenitrile (8ba). A pale yellow oil, $\mathrm{R}_{\mathrm{f}} 0.18$ (hexane-ethyl acetate $\left.=50: 1\right) .{ }^{1} \mathrm{H} \mathrm{NMR}\left(400 \mathrm{MHz}, \mathrm{CDCl}_{3}\right) \delta 2.16(\mathrm{t}, J=7.5$ $\mathrm{Hz}, 2 \mathrm{H}), 2.08$ (t, $J=7.8 \mathrm{~Hz}, 2 \mathrm{H}), 2.02(\mathrm{~s}, 2 \mathrm{H}), 1.56$ (sext, $J=7.5 \mathrm{~Hz}, 2 \mathrm{H}), 1.44$ (sext, $J=7.6 \mathrm{~Hz}, 2 \mathrm{H}), 0.95(\mathrm{t}, J=7.3 \mathrm{~Hz}, 3 \mathrm{H}), 0.94(\mathrm{t}, J=7.3 \mathrm{~Hz}, 3 \mathrm{H}), 0.11(\mathrm{~s}, 9 \mathrm{H})$; ${ }^{13} \mathrm{C}$ NMR $\left(101 \mathrm{MHz}, \mathrm{CDCl}_{3}\right) \delta 159.1,120.4,105.7,35.7,31.4,28.8,22.3,21.6,14.2,13.6,-0.6$; IR (neat): 2961, 2874, 2203, 1611, 1464, 1421, 1379, 1250, 1148, 1078, 853, 766, $694 \mathrm{~cm}^{-1}$; Anal. Calcd for $\mathrm{C}_{13} \mathrm{H}_{25} \mathrm{NSi}$; C, 69.88; H, 11.28. Found: C, 70.18; H, 11.17.

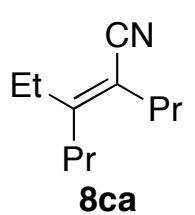

(Z)-3-Ethyl-2-propyl-2-hexenenitrile (8ca). A colorless oil, $\mathrm{R}_{\mathrm{f}} 0.18$ (hexane-ethyl acetate $=40: 1) .{ }^{1} \mathrm{H}$ NMR $\left(400 \mathrm{MHz}, \mathrm{CDCl}_{3}\right) \delta 2.40(\mathrm{q}, J=7.5 \mathrm{~Hz}, 2 \mathrm{H}), 2.17(\mathrm{t}, J=7.3 \mathrm{~Hz}$, 2H), 2.15 (t, $J=7.9 \mathrm{~Hz}, 2 \mathrm{H}), 1.57$ (sext, $J=7.4 \mathrm{~Hz}, 2 \mathrm{H}$ ), 1.43 (sext, $J=7.6 \mathrm{~Hz}, 2 \mathrm{H}$ ), 1.09 $(\mathrm{t}, J=7.6 \mathrm{~Hz}, 3 \mathrm{H}), 0.944(\mathrm{t}, J=7.3 \mathrm{~Hz}, 3 \mathrm{H}), 0.940(\mathrm{t}, J=7.3 \mathrm{~Hz}, 3 \mathrm{H}) ;{ }^{13} \mathrm{C} \mathrm{NMR}(101$ $\mathrm{MHz}_{\mathrm{CDCl}}$ ) $\delta 161.1,119.1,109.2,33.0,31.5,29.3,21.9,21.4,14.3,13.6,13.2$; IR (neat): 2964, 2936, 2874, 2208, 1624, 1464, 1379, 1138, 1057, 797, $741 \mathrm{~cm}^{-1}$; Anal. Calcd for $\mathrm{C}_{11} \mathrm{H}_{19} \mathrm{~N}$; C, 79.94; H, 11.59. Found: C, 79.91; H, 11.71.

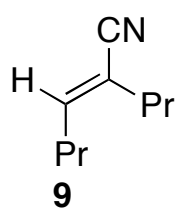

(E)-2-Propyl-2-hexenenitrile (9). A colorless oil, $\mathrm{R}_{\mathrm{f}} 0.18$ (hexane-ethyl acetate $=40: 1$ ). ${ }^{1} \mathrm{H}$ NMR (400 MHz, $\mathrm{CDCl}_{3}$ ) $\delta 6.35$ (t, $J=7.6 \mathrm{~Hz}, 1 \mathrm{H}$ ), 2.17 (quint, $J=7.5 \mathrm{~Hz}, 4 \mathrm{H}$ ), 1.58 (sext, $J=7.4 \mathrm{~Hz}, 2 \mathrm{H}), 1.46(\mathrm{sext}, J=7.4 \mathrm{~Hz}, 2 \mathrm{H}) 0.95$ (t, $J=7.4 \mathrm{~Hz}, 3 \mathrm{H}), 0.94$ (t, $J=7.4$ $\mathrm{Hz}, 3 \mathrm{H}) ;{ }^{13} \mathrm{C} \mathrm{NMR}\left(101 \mathrm{MHz}, \mathrm{CDCl}_{3}\right) \delta 147.9,120.1,114.8,30.5,30.4,21.9,21.4,13.8$, 13.5; IR (neat): 2963, 2934, 2874, 2216, 1634, 1460, 1381, 1067, $908 \mathrm{~cm}^{-1}$; HRMS (EI) Calcd for $\mathrm{C}_{9} \mathrm{H}_{15} \mathrm{~N}: \mathrm{M}^{+}, 137.1204$. Found: $m / z$ 137.1209. 


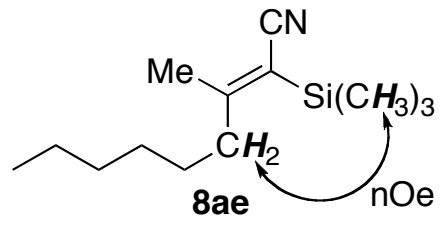

(Z)-3-Methyl-2-trimethylsilyl-2-nonenenitrile (8ae). A colorless oil, $\mathrm{R}_{\mathrm{f}}$ 0.15 (hexane-ethyl acetate $=30: 1) .{ }^{1} \mathrm{H}$ NMR $\left(400 \mathrm{MHz}, \mathrm{CDCl}_{3}\right) \delta 2.25(\mathrm{t}$, $J=8.0 \mathrm{~Hz}, 2 \mathrm{H}), 2.15(\mathrm{~s}, 3 \mathrm{H}), 1.50-1.40(\mathrm{~m}, 2 \mathrm{H}), 1.37-1.24(\mathrm{~m}, 6 \mathrm{H}) 0.90(\mathrm{t}$, $J=6.9 \mathrm{~Hz}, 3 \mathrm{H}), 0.28(\mathrm{~s}, 9 \mathrm{H}) ;{ }^{13} \mathrm{C} \mathrm{NMR}\left(101 \mathrm{MHz}, \mathrm{CDCl}_{3}\right) \delta 174.2,120.3$, 108.7, 38.7, 31.7, 29.5, 28.4, 24.4, 22.6, 14.1, 0.08; IR (neat): 2957, 2930, 2858, 2197, 1583, 1466, 1377, 1254, 843, $762 \mathrm{~cm}^{-1}$; Anal. Calcd for $\mathrm{C}_{13} \mathrm{H}_{25} \mathrm{NSi}$; C, 69.88; H, 11.28. Found: C, 69.81; H, 11.42.

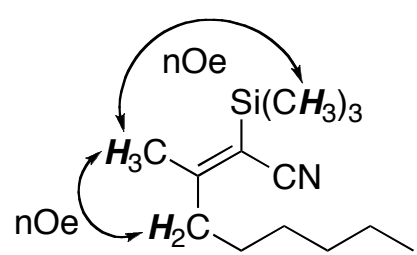

$(E)-8 \mathrm{ae}$

(E)-3-Methyl-2-trimethylsilyl-2-nonenenitrile [(E)-8ae]. A colorless oil, $\mathrm{R}_{\mathrm{f}} 0.15$ (hexane-ethyl acetate $\left.=30: 1\right) .{ }^{1} \mathrm{H}$ NMR $\left(400 \mathrm{MHz}, \mathrm{CDCl}_{3}\right) \delta 2.49(\mathrm{t}$, $J=7.8 \mathrm{~Hz}, 2 \mathrm{H}), 1.97(\mathrm{~s}, 3 \mathrm{H}), 1.55-1.46(\mathrm{~m}, 2 \mathrm{H}), 1.39-1.26(\mathrm{~m}, 6 \mathrm{H}) 0.89(\mathrm{t}$, $J=6.9 \mathrm{~Hz}, 3 \mathrm{H}), 0.29(\mathrm{~s}, 9 \mathrm{H}) ;{ }^{13} \mathrm{C} \mathrm{NMR}\left(101 \mathrm{MHz}, \mathrm{CDCl}_{3}\right) \delta 174.2,120.0$, 108.3, 41.0, 31.7, 29.1, 28.1, 22.63, 22.56, 14.2, -0.2; IR (neat): 2957, 2930, 2858, 2195, 1583, 1462, 1377, 1254, 843, $760 \mathrm{~cm}^{-1}$; Anal. Calcd for $\mathrm{C}_{13} \mathrm{H}_{25} \mathrm{NSi}$; C, 69.88; H, 11.28. Found: C, 69.98; H, 11.08 .

\section{References}

1. Pangborn, A. B.; Giardello, M. A.; Grubbs, R. H.; Rosen, R. K.; Timmers, F. J. Organometallics 1996, 15, 1518-1520.

2. Nakao, Y.; Chen, J.; Imanaka, H.; Hiyama, T. submitted for publication.

3. Hartwig, J. F.; Kawatsura, M.; Hauck, S. I.; Shaughnessy, K. H.; Alcazar-Roman, L. M. J. Org. Chem. 1999, 64, 5575-5580.

4. Nakao, Y.; Kanyiva, K. S.; Oda, S.; Hiyama, T. J. Am. Chem. Soc. 2006, 128, 8146-8147.

5. Buckle, D. R.; Rockell, C. J. M. J. Chem. Soc., Perkin Trans. I 1985, 11, 2443-2446.

6. Dahl, O. Acta Chem. Scand. 1969, 23, 2342-2354.

7. Wu, K.; Midland, M. M.; Okamura, W. H. J. Org. Chem. 1990, 55, 4381-4392.

8. Bent, A.; Blommaert, A. G. S.; Melman, C. T. M.; IJzerman, A.P.; Wijngaarden, I.; Soudijn, W. J. Med. Chem. 1992, 35, 1042-1049.

9. Ashby, E. C.; Noding, S. A. J. Org. Chem. 1980, 45, 1035-1041.

10. Nakao, Y.; Oda, S.; Yada, A.; Hiyama, T. Tetrahedron 2006, 62, 7567-7576. 

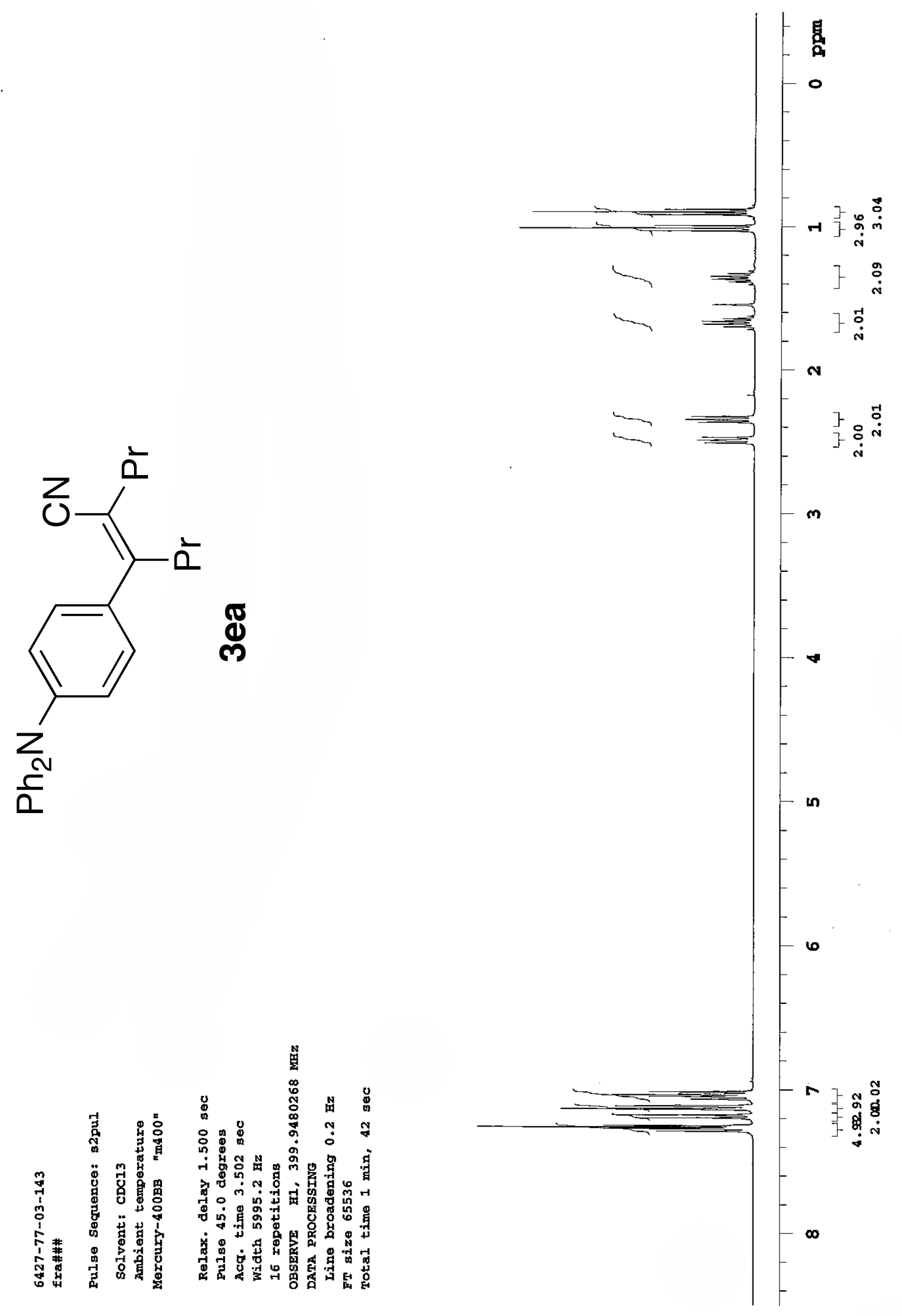


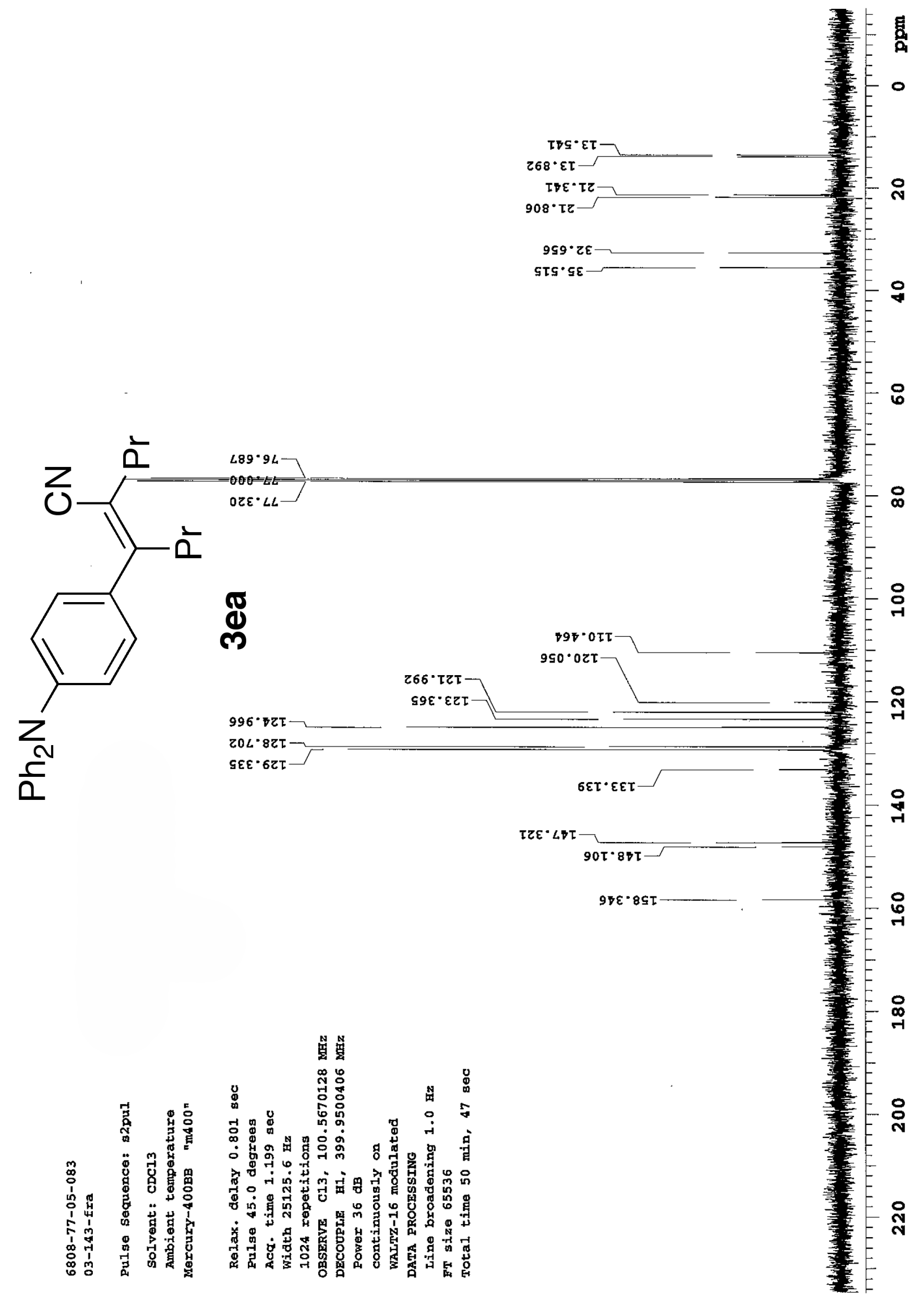



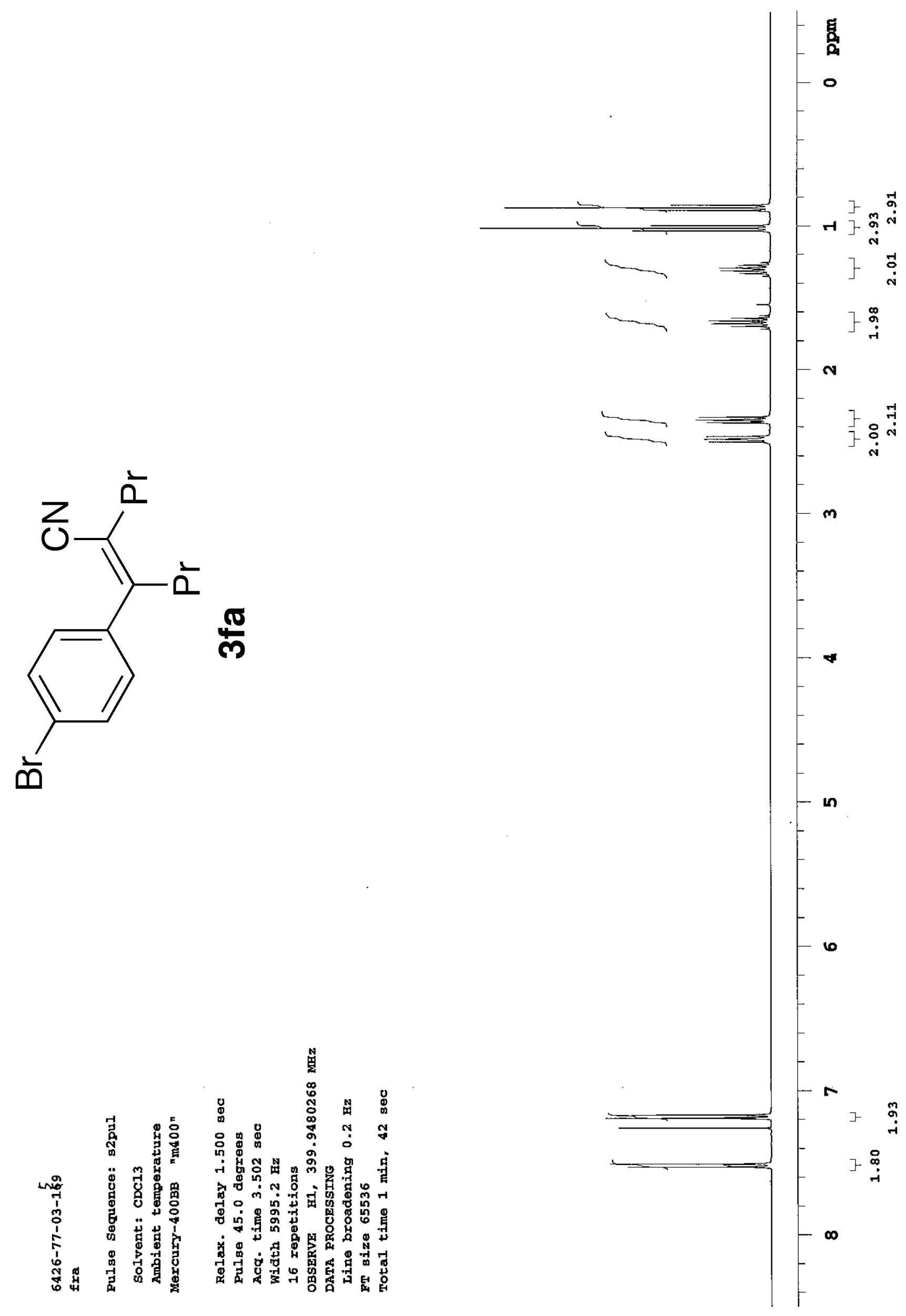


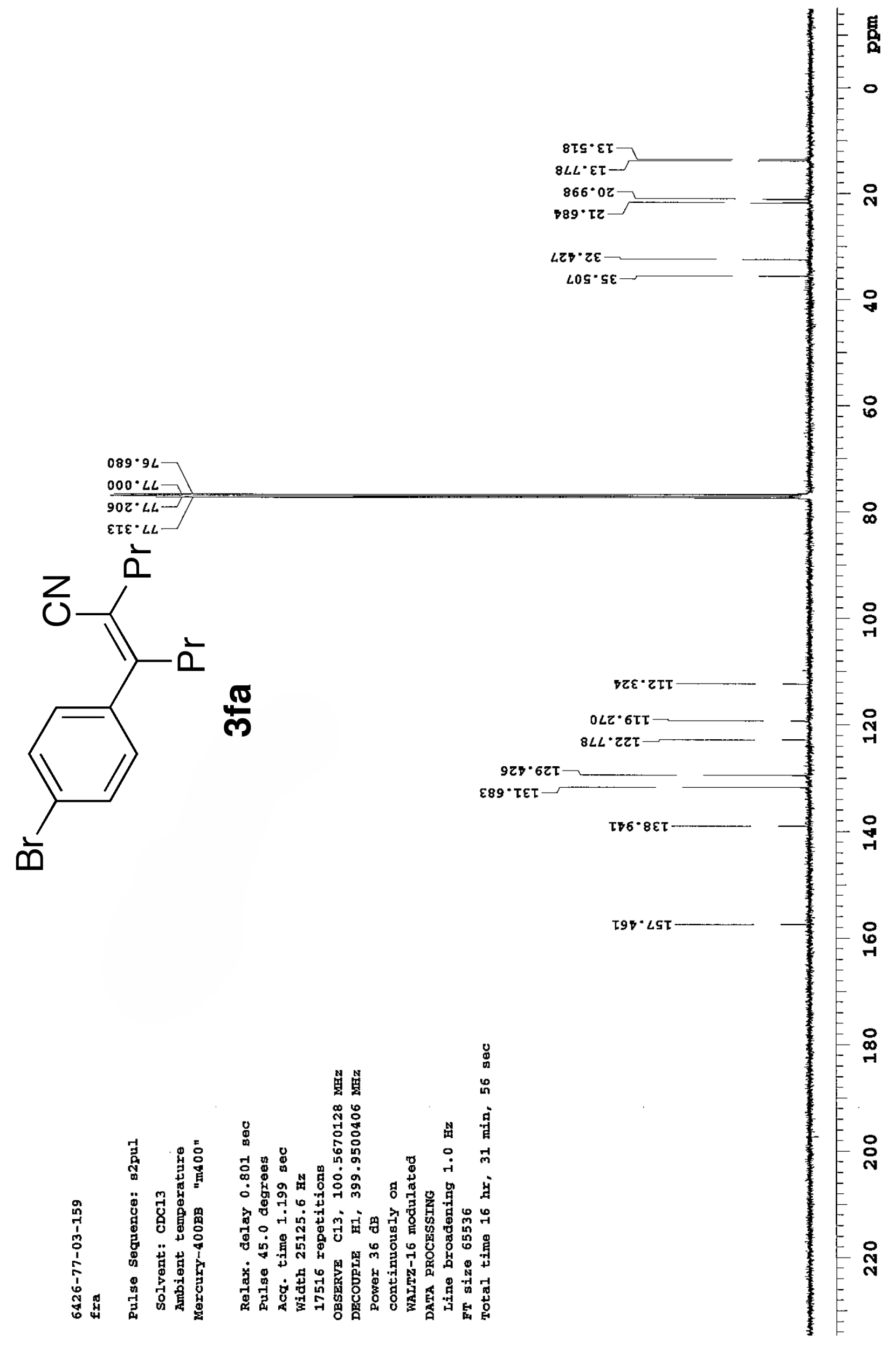



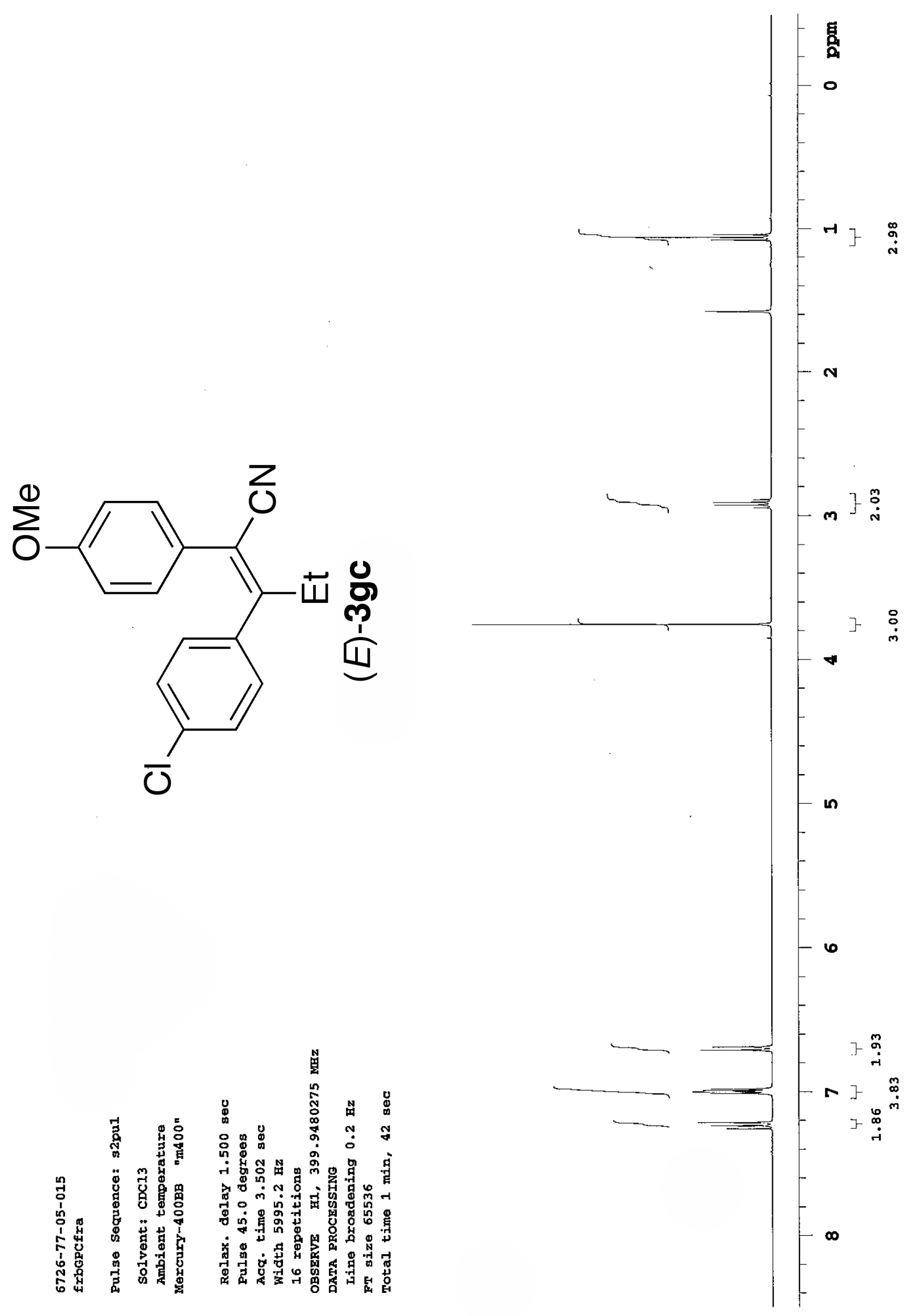


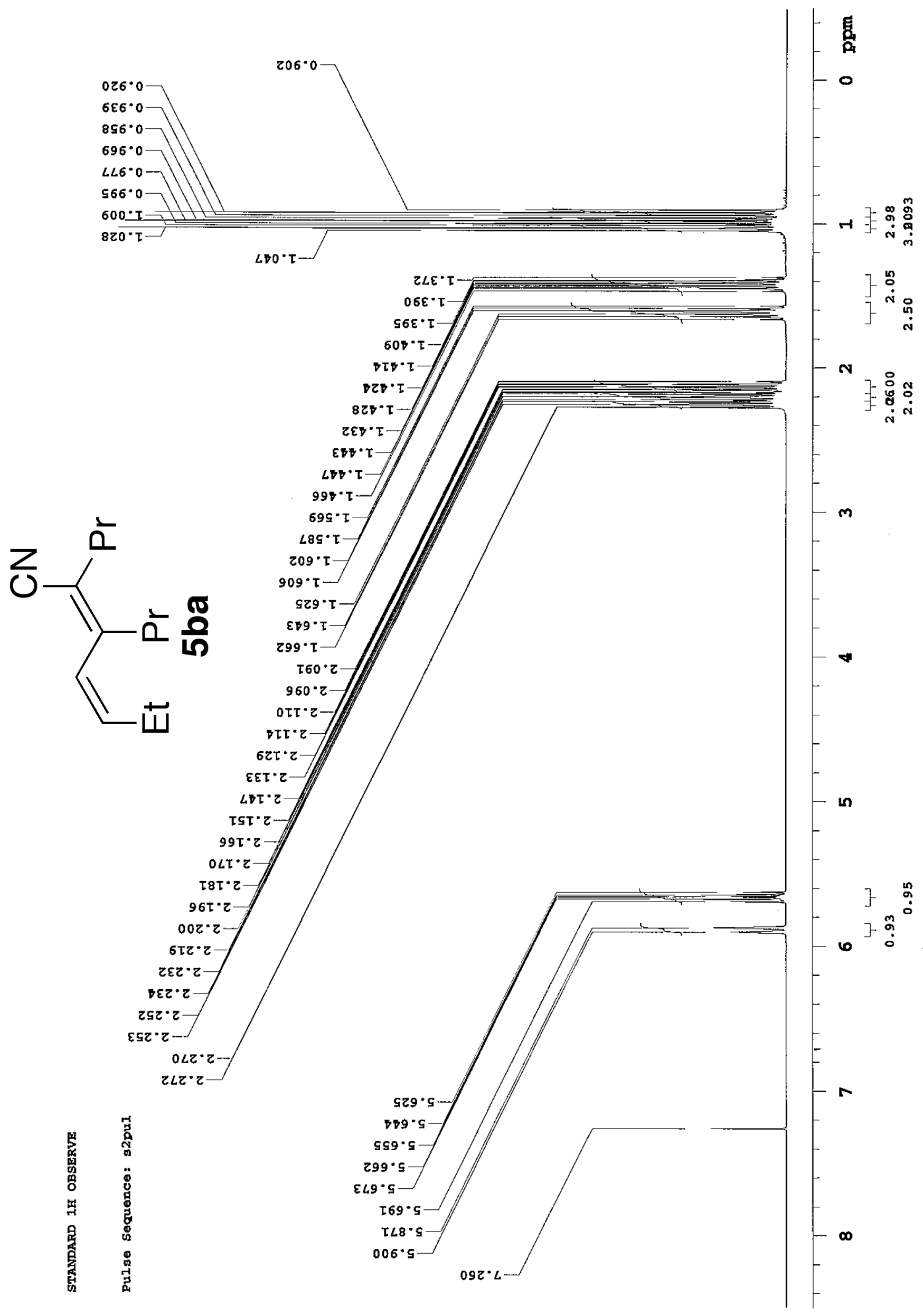




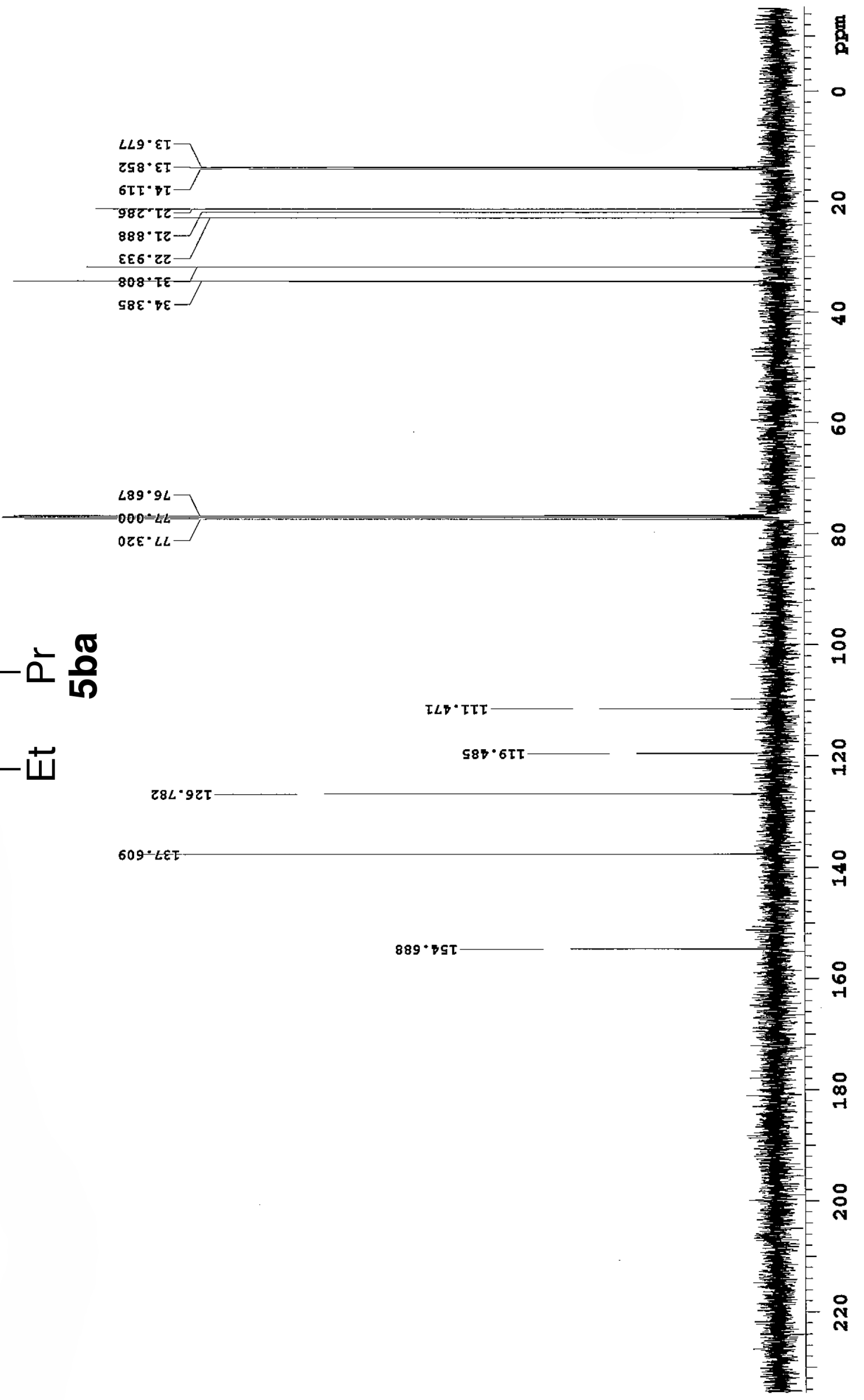




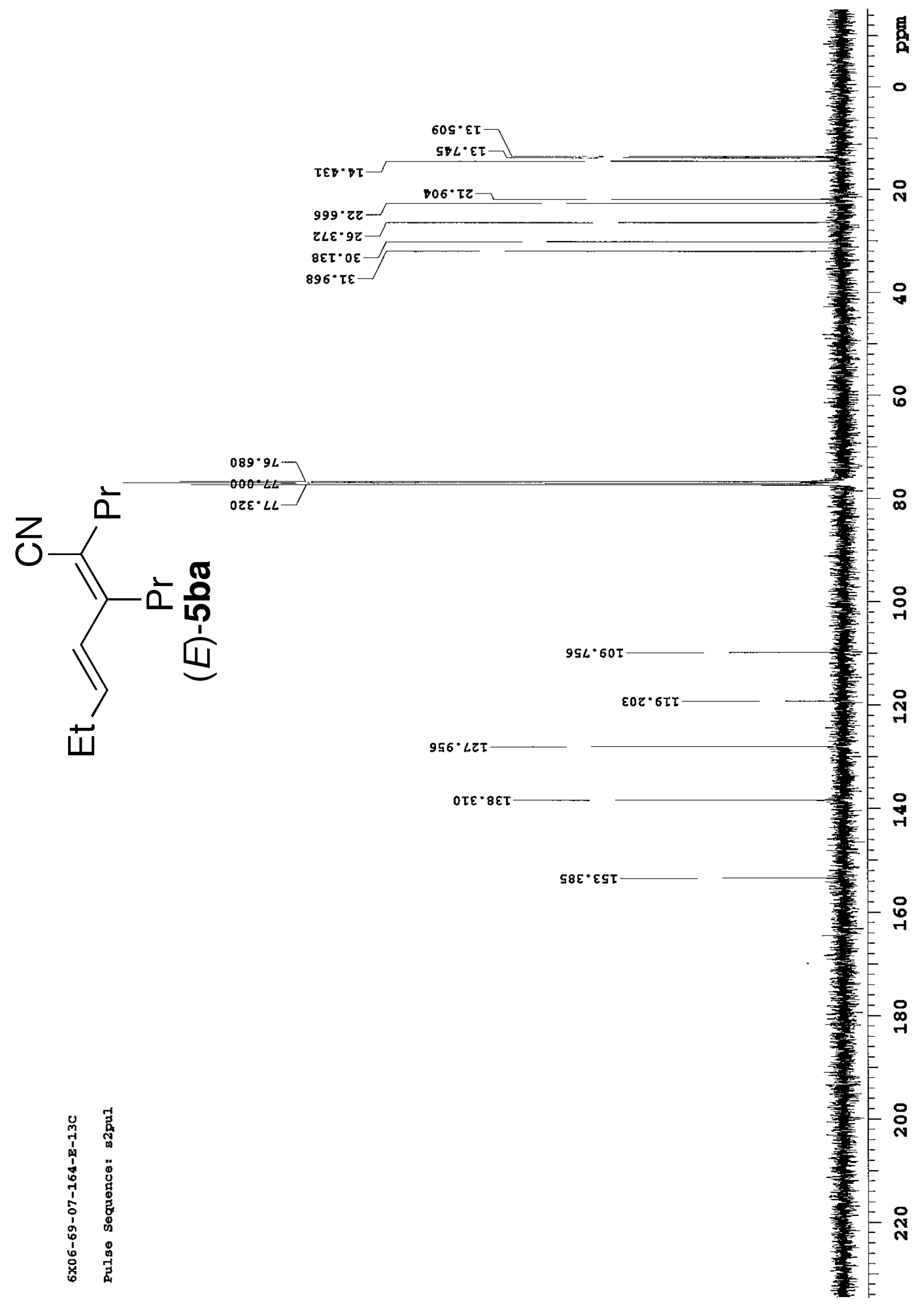




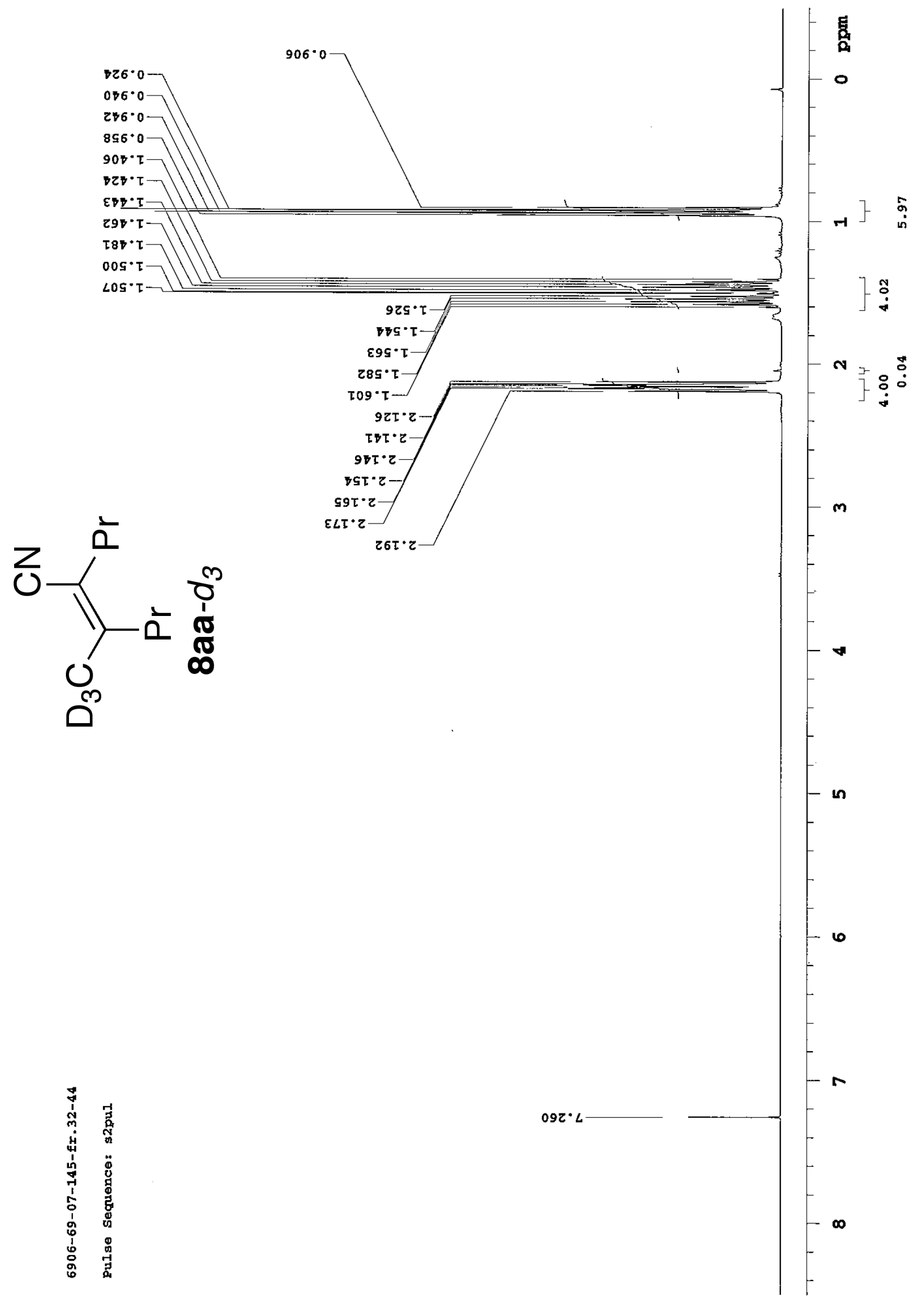




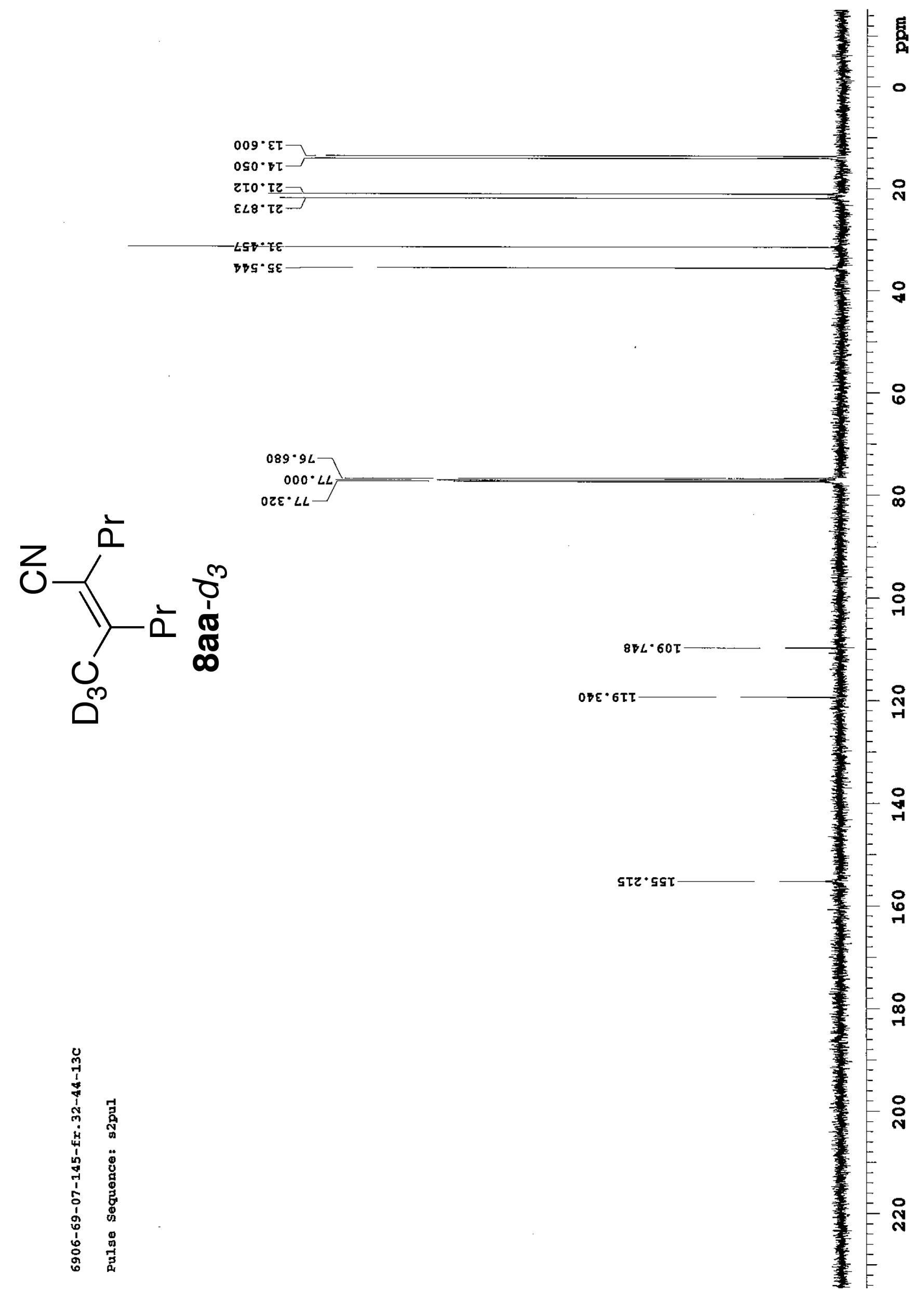



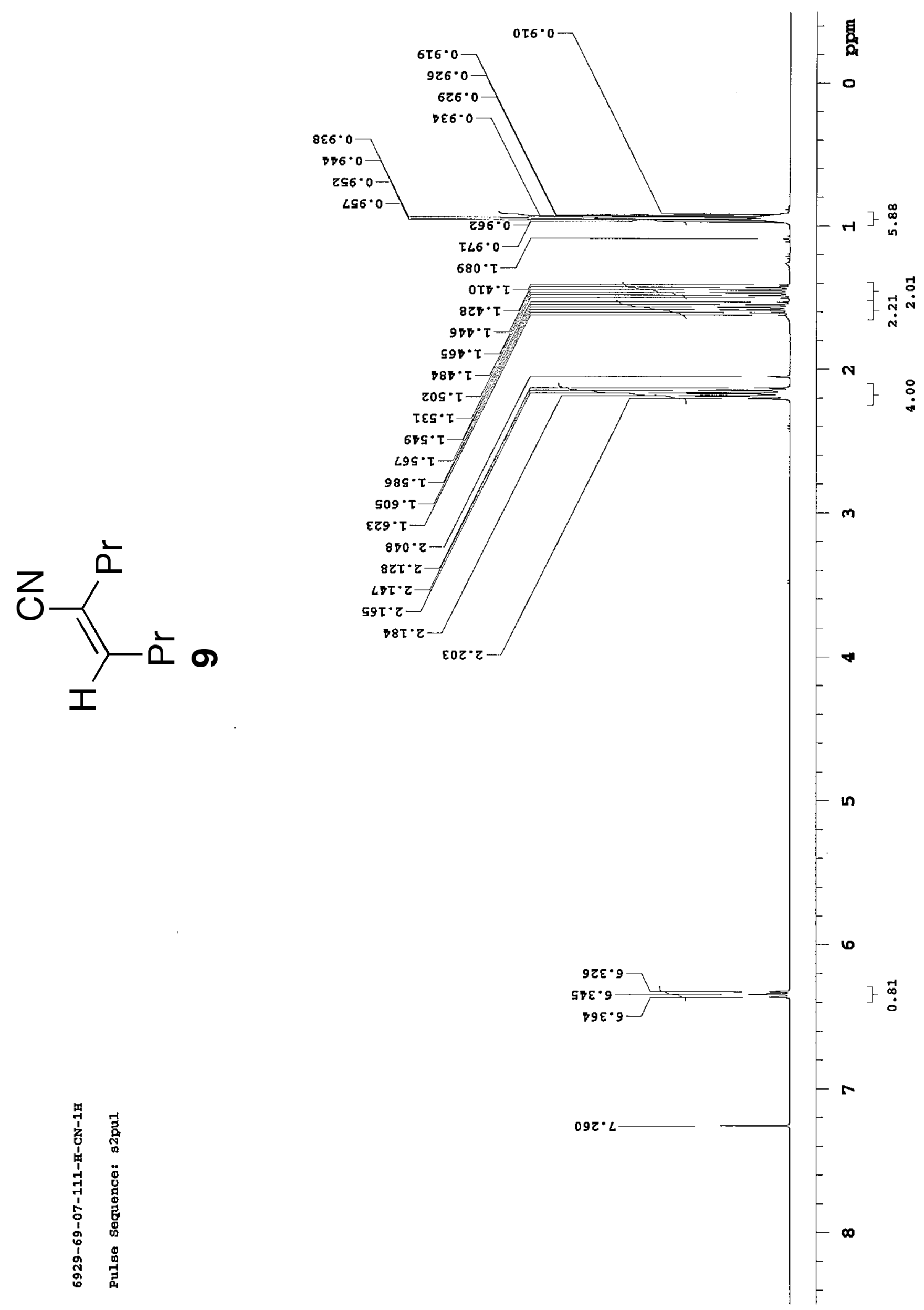


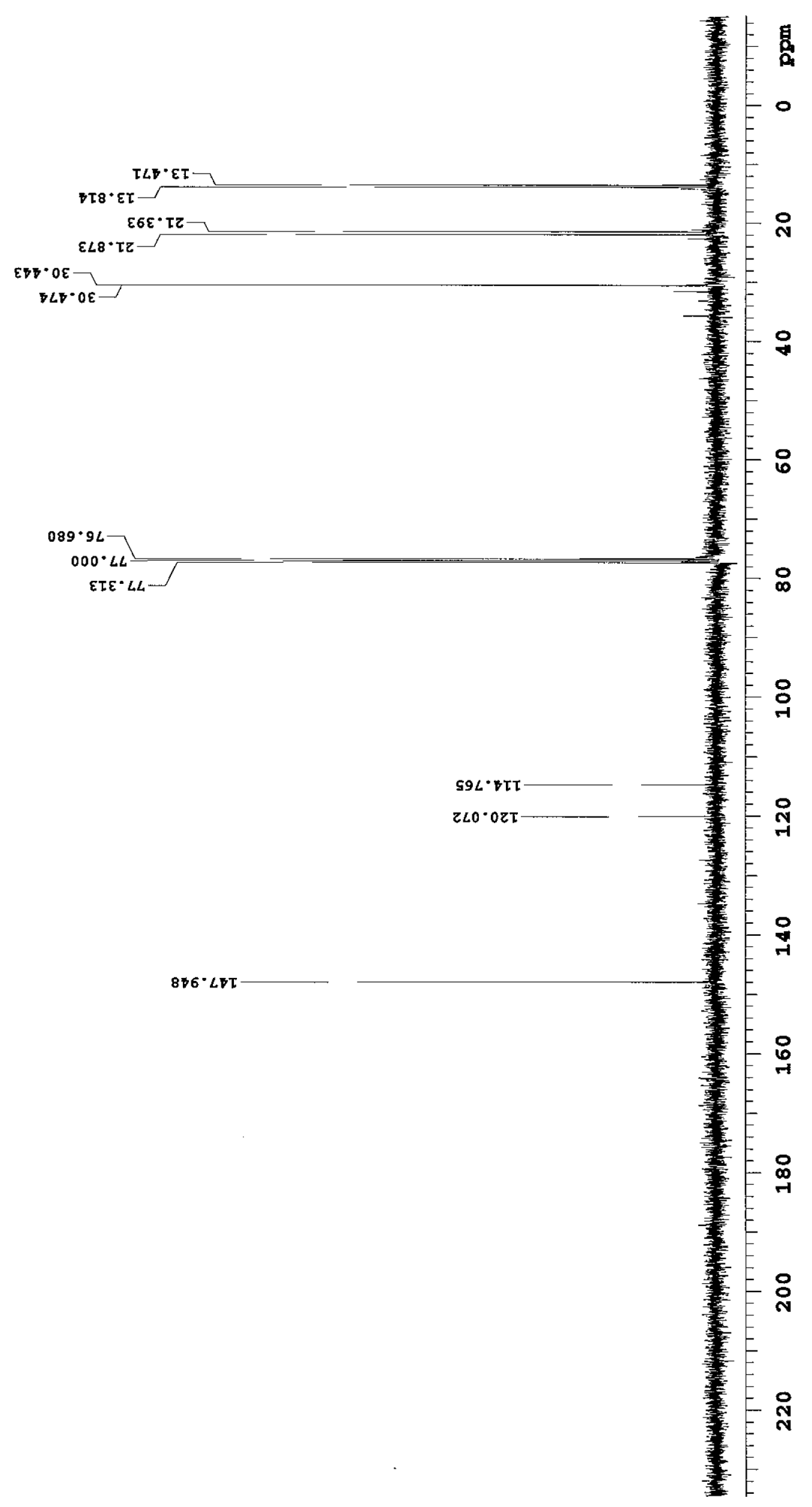

